\title{
Constraints on the fundamental topological parameters of spatial tessellations
}

\author{
Richard Cowan \\ School of Mathematics and Statistics \\ University of Sydney, NSW, 2006, Australia \\ e-mail: rcowan@usyd.edu.au \\ Viola Weiss \\ Ernst-Abbe-Fachhochschule Jena \\ D-07703 Jena, Germany. \\ e-mail:Viola.Weiss@fh-jena.de
}

\begin{abstract}
Tessellations of $\mathbb{R}^{3}$ that use convex polyhedral cells to fill the space can be extremely complicated, especially if they are not 'facet-to-facet', that is, if the facets of a cell do not necessarily coincide with the facets of that cell's neighbours. In a recent paper [15], we have developed a theory which covers these complicated cases, at least with respect to their combinatorial topology. The theory required seven parameters, three of which suffice for facet-to-facet cases; the remaining four parameters are needed for the awkward adjacency concepts that arise in the general case. This current paper establishes constraints that apply to these seven parameters and so defines a permissible region within their sevendimensional space, a region which we discover is not bounded. Our constraints in the relatively simple facet-to-facet case are also new.
\end{abstract}

Key words: random geometry, tessellations, tilings, packing of polyhedra, space-filling, combinatorial topology, cell complex, parameter constraints.

MSC (2010): Primary: 60D05; 05B 45; 52C17

Secondary: 60G55; 51M20; 52B10

\section{Introduction}

In this paper we continue our study of random stationary spatial tessellations, that is, random tessellations of the three-dimensional space $\mathbb{R}^{3}$ having statistical properties that are invariant under translation. In [15], we developed a theory of such tessellations in cases where the cells of the tessellation are closed convex polyhedra and not necessarily facet-to-facet. Seven parameters were needed to address the major topological issues.

Primitive elements: Three of these parameters suffice for the relatively simple facet-tofacet case, where the questions of interest focus on the four primitive elements of the tessellation, vertices, edges, plates and cells. (We use the word plate for a closed convex polygon which lies on the boundary of two cells; if these two closed cells are $z$ and $z^{\prime}$, then the polygon $z \cap z^{\prime}$ is that plate.) The three parameters, with cyclic subscripts in the letters $V, E$ and $P$, are:

- $\mu_{V E}$, the expected number of edges emanating from the typical vertex;

- $\mu_{E P}$, the expected number of plates emanating from a typical edge;

- $\mu_{P V}$, the expected number of vertices lying on the boundary of the typical polygonal plate. Note that if a plate is an $n$-gon, this number may exceed $n$ in the non facet-to-facet case because plate corners are not the same entities as tessellation vertices. 
As a matter of notation, classes of the primitive elements vertices, edges, plates and cells are called $V, E, P$ and $Z$. The generic expression, $m_{Y}(x)$ for $x \in X$, is defined as the number of objects of type $Y$ adjacent to a particular $x$ in the object class $X$. We define $\mu_{X Y}$ as the expected number of $Y$-type objects adjacent to the typical object of class $X$. Two objects $x$ and $y$ are adjacent if either $x \subseteq y$ or $y \subseteq x$. Adjacency is a precise concept which covers relationships like 'emanating from' or 'lying on', used above. All twelve mean adjacencies $\mu_{X Y}$, for $X$ and $Y$ primitive, $X \neq Y$, can be expressed (see Table 1, proved in [15]) as functions of the cyclic adjacency parameters, $\mu_{V E}, \mu_{E P}$ and $\mu_{P V}$.

From [15], we reproduce the following table. It uses the abbreviation,

$$
f(x):=\mu_{V E} \mu_{E P}-x\left(\mu_{V E}-2\right) .
$$

\begin{tabular}{|c||c|}
\hline$X$ & $\lambda_{X} / \lambda_{V}$ \\
\hline \hline vertices $V$ & 1 \\
edges $E$ & $\frac{1}{2} \mu_{V E}$ \\
plates $P$ & $\frac{\mu_{V E} \mu_{E P}}{2 \mu_{P V}}$ \\
cells $Z$ & $\frac{f\left(\mu_{P V}\right)}{2 \mu_{P V}}$ \\
\hline
\end{tabular}

\begin{tabular}{|c||c|c|c|c|}
\hline$\mu$ & $V$ & $E$ & $P$ & $Z$ \\
\hline \hline$V$ & 1 & $\mu_{V E}$ & $\frac{1}{2} \mu_{V E} \mu_{E P}$ & $\frac{1}{2} f(2)$ \\
$E$ & 2 & 1 & $\mu_{E P}$ & $\mu_{E P}$ \\
$P$ & $\mu_{P V}$ & $\mu_{P V}$ & 1 & 2 \\
$Z$ & $\frac{\mu_{P V} f(2)}{f\left(\mu_{P V}\right)}$ & $\frac{\mu_{V E} \mu_{E P} \mu_{P V}}{f\left(\mu_{P V}\right)}$ & $\frac{2 \mu_{V E} \mu_{E P}}{f\left(\mu_{P V}\right)}$ & 1 \\
\hline
\end{tabular}

Table 1: Primitive intensities and adjacencies, using the abbreviation $f$, defined above in (1). All entries in the table are expressed in terms of the scale parameter, $\lambda_{V}$, and the cyclic three mean-adjacencies. Note that there are three linear identities within the table: $\lambda_{V}-\lambda_{E}+\lambda_{P}-\lambda_{Z}=0 ; \mu_{V E}-\mu_{V P}+\mu_{V Z}=2 ; \mu_{Z V}-\mu_{Z E}+\mu_{Z P}=2$. These have links with Euler's polyhedral formula and with related formulae from cell-complex theory ([5], [16]).

The intensity of objects of type $X$ (mean number of centroids per unit volume) is denoted by $\lambda_{X}$. The value of $\lambda_{V}$ determines the scale of the tessellation and in Table 1 the three others, $\lambda_{E}, \lambda_{P}$ and $\lambda_{Z}$, are also expressed in terms of $\lambda_{V}$ and the cyclic adjacencies, $\mu_{V E}, \mu_{E P}$ and $\mu_{P V}$.

Subsets of the generic class $X$ are denoted by $X[\cdot]$, with a suitably chosen symbol in the square brackets. For example, shortly we introduce a subclass of edges known as $\pi$-edges; we use the notation $E[\pi]$ for this subclass.

Adjacency is a symmetric relationship and an important identity applies, proved in Møller's Theorem 5.1 [8] and discussed as equation (5) in [15].

$$
\lambda_{X} \mu_{X Y}=\lambda_{Y} \mu_{Y X}
$$

Although the results in Table 1 have been known for some time, at least since 1980 (see $[12]$ ), there have been no studies of the constraints which apply to the three mean-adjacencies, $\mu_{V E}, \mu_{E P}$ and $\mu_{P V}$ (even in the facet-to-facet situation). We shall rectify this deficiency in this paper. After presenting some examples of spatial tessellations in Section 2, we then focus on facet-to-facet cases and show, in Section 3, that:

$$
4 \leq \mu_{V E} ; \quad 3 \leq \mu_{E P} \leq 6\left(1-\frac{2}{\mu_{V E}}\right)<6 ; \quad 3 \leq \mu_{P V}<\frac{\mu_{V E} \mu_{E P}}{\mu_{V E}-2} \leq 6 .
$$

So these constraints, which are derived using the information in Table 1 combined with elementary geometry of convex polyhedra, apply to all facet-to-facet tessellations. In Section 4, we 


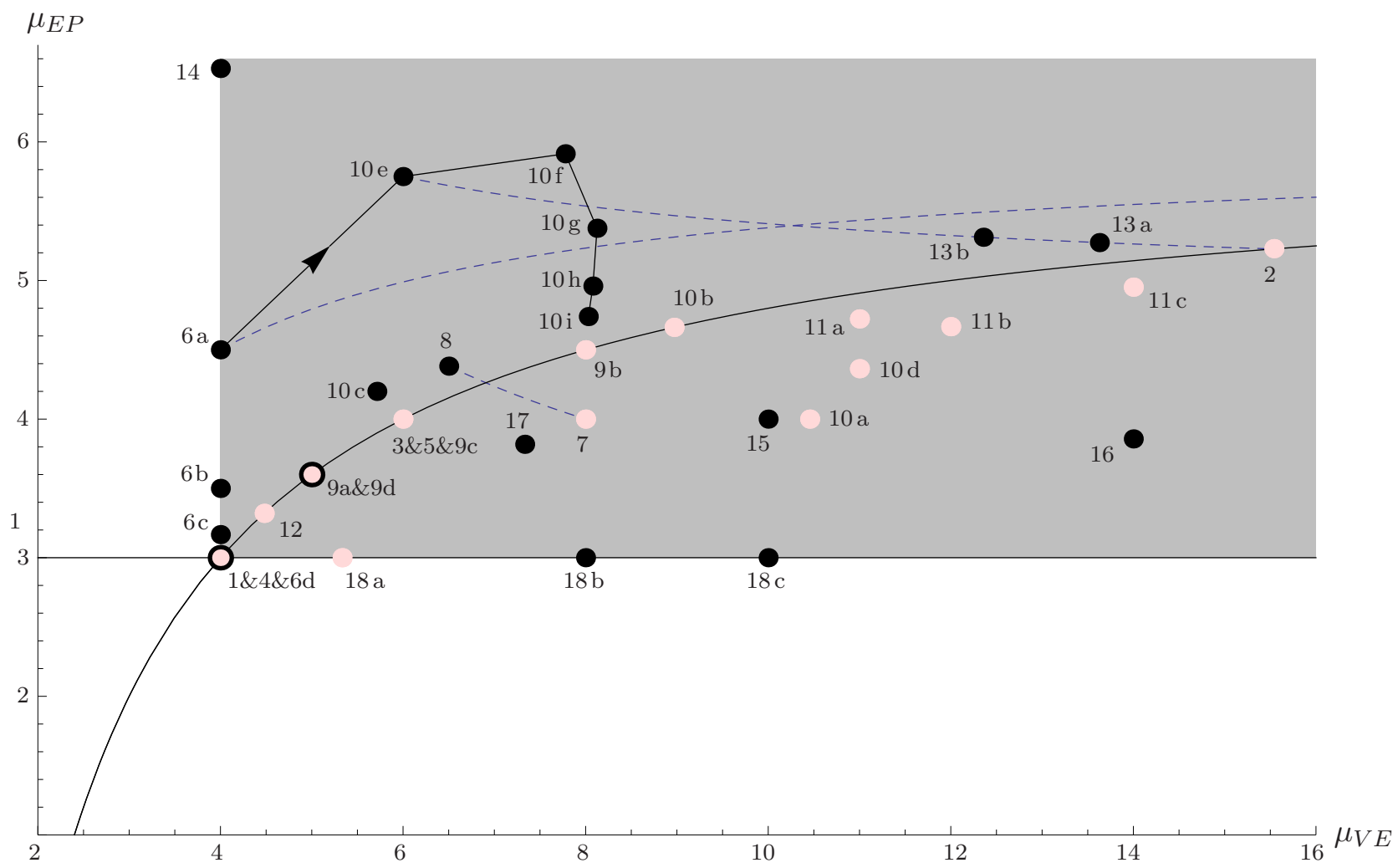

Figure 1: A plot of $\mu_{E P}=6\left(1-2 / \mu_{V E}\right)$, shown as the solid smooth curve, together with $\left(\mu_{V E}, \mu_{E P}\right)$ values for the various models discussed in this paper. Facet-to-facet models have a light-coloured dot and non facet-to-facet models a black dot. Two of the dots are hybrid dots; the $(4,3)$ dot represents one facet-to-facet model (Example 1 ) and two that are not (Examples 4 and $6 \mathrm{~d}$ ) and, for the $(5,18 / 5)$ dot, Example 9a is facet-to-facet whilst $9 \mathrm{~d}$ is not. Dashed curves and the connected sequence of line segments joining some of the dots are explained in the text.

present more facet-to-facet examples, including one construction in which $\mu_{V E}$ can be arbitrary large. Thus we reinforce the absence of an upper bound for $\mu_{V E}$ in (3).

The examples throughout our paper enable us to overlay data points $\left(\mu_{V E}, \mu_{E P}\right)$ on a plot of the curve $\mu_{E P}=6\left(1-2 / \mu_{V E}\right)$, revealing the role that the middle constraint in (3) plays. We shall discover, as illustrated in Figure 1, that one can find some non facet-to-facet examples where (3) also holds - though for many such examples (3) is violated.

The dashed curves in Figure 1 result from mixtures of tessellations; these are explained in Section 5. The dots which are linked by a connected sequence of line segments are explained in Example 10.

Faces of the primitive elements: Section 6 commences the theory for tessellations which are not facet-to-facet. It presents a different set of constraints for $\mu_{V E}, \mu_{E P}$ and $\mu_{P V}$ as well as constraints on the four additional parameters, $\xi, \kappa, \psi$ and $\tau$. These four parameters come from our earlier study [15] and quantify the consequences of having cell-facets that do not coincide with those of their neighbours.

To define these parameters, we need to settle the terminology for faces of the primitive elements - in view of the fact that the standard terminologies in tessellation and polytope theories clash. We have decided to use the tessellation theoretic meaning of words vertex, edge, plate and cell; other names, apex, ridge and facet are used for the $j$-faces of a cell, $j=0,1,2$. A $\mathrm{j}$-face of a polygon $(\mathrm{j}=0,1)$ we call a corner or side. We distinguish between sides of plates and of facets by using the terms plate-side and facet-side. 
We note that in a facet-to-facet tessellation every face of a primitive element is equal (as a set in $\mathbb{R}^{3}$ ) to a primitive element. For example, every ridge (1-face of a cell) equals an edge of the tessellation - and every edge equals at least three ridges. This simplicity is lost in the non facet-to-facet case.

Let $X$ be a class of convex polytopes, each member of the class having dimension $i \leq 3$. We define $X_{j}, j<i$, as the class of objects which are $j$-dimensional faces ( $j$-faces) of some polytope belonging to $X$. For instance $P_{1}$ is the class of the plate-sides (1-faces of plates) and $Z_{2}$ is the class of facets (2-faces of cells). We denote the expected number of $j$-faces that a typical polytope of class $X$ has by $\nu_{j}(X)$. For example, $\nu_{1}(Z)$ is the expected number of ridges (1-faces of the cells) for a typical cell. Adjacency formulae and intensities involving these classes of elements were derived in our earlier paper [15] and will be introduced in Section 6 as needed. Section 2 of that paper also has a more detailed discussion of our face-of-polytope classes, which may in some cases be multisets.

The four interior parameters, $\xi, \kappa, \psi$ and $\tau$ : The primitive elements, edge, plate and cell, can have no other element (or face of an element) lying in their relative interior. The face of a primitive element, however, can have such interior structure - and the four interior parameters quantify the prevalence of this phenomenon in non facet-to-facet tessellations. These additional parameters introduced in [15] and visualised in Figure 2] (and especially in Figure 3 of [15] in the context of elaborate cell architecture) are defined as follows. We use the notation where $\stackrel{\circ}{X}$ means the class of relative interiors of members of $X$, and where the word 'interior' will henceforth mean 'relative interior'.

- $\xi:=$ the proportion of edges in the tessellation whose interior is contained in the interior of some facet - these being called $\pi$-edges in [15]. Thus $\xi=\lambda_{E[\pi]} / \lambda_{E}$. Note also that an adjacency representation exists, namely $\xi=\mu_{E Z_{2}}^{\circ}$.

- $\kappa:=$ the proportion of vertices in the tessellation contained in the interior of some facet - these being called hemi-vertices because half of the neighbourhood of such a vertex is a hemi-sphere lying within one cell. Thus $\kappa=\lambda_{V[h e m i]} / \lambda_{V}$. Note also that $\kappa=\mu_{V Z_{2}}$.

- $\psi:=\mu_{V Z_{1}}$, the expected number of ridge-interiors adjacent to a typical vertex.

- $\tau:=\mu_{V} \stackrel{\circ}{P_{1}}$, the expected number of plate-side-interiors adjacent to a typical vertex.

Being proportions, $\xi$ and $\kappa$ must lie in the unit interval, so $(\kappa, \xi) \in[0,1]^{2}$. We note that the first of these four parameters, $\xi$, is a descriptor of edges, whilst the last three describe vertices. It is possible however to write $\xi$ in terms of the vertex adjacency, $\mu_{V E[\pi]}$. Using (2), Table 1 and the fact that $\mu_{E[\pi] V}=2$,

$$
\xi:=\frac{\lambda_{E[\pi]}}{\lambda_{E}}=\frac{\lambda_{E[\pi]} \mu_{E[\pi] V}}{2 \lambda_{E}}=\frac{\lambda_{V} \mu_{V E[\pi]}}{2 \lambda_{E}}=\frac{\mu_{V E[\pi]}}{\mu_{V E}} .
$$

Later we use this linkage between the vertex property $\mu_{V E[\pi]}$, the mean number of $\pi$-edges adjacent to a typical vertex, and the proportion $\xi$ of $\pi$-edges.

The relationship with cell complex theory: In the theory of cell complexes the terminology face-to-face has been used (see [13], p.447). In the context of spatial tessellations, this means the following: if the intersection of two cells has dimension $j \in\{0,1,2\}$, then the intersection is a $j$-face of both cells. We have chosen to use the concept facet-to-facet, which has the same meaning but apparently only when $j=2$. The following lemma provides an equivalence of these two concepts. 
LEMMA 1: Our concept of facet-to-facet is equivalent to the concept of face-to-face used in the literature of cell complexes.

ProOF: Clearly, face-to-face implies facet-to-facet, so we focus on the converse to this.

Firstly, being facet-to-facet is equivalent to having $\xi=0$, because $\xi>0 \Longleftrightarrow$ the existence of $\pi$-edges and having $\pi$-edges would violate the condition that every facet of a cell $z$ coincides with the facet of one of its neighbouring cells, for all $z \in Z$.

Secondly, $\xi=0$ implies that $\kappa=\psi=\tau=0$. This follows because hemi-vertices, and vertices which lie in the interior of a ridge or a plate-side, have emanating $\pi$-edges. If there are no $\pi$-edges, there can be none of these vertices.

Thirdly, the condition $\xi=\kappa=\psi=\tau=0$ is equivalent to being face-to-face. This equivalence can be understood by noting that the face-to-face condition prevents a $j$-face of a cell $z$ being contained in the interior of a $k$-face $(0 \leq j<k \leq 2)$ of any other cell $z^{\prime}$ (because $z \cap z^{\prime}$ would be of dimension $j$ but not a $j$-face of $z^{\prime}$.) Furthermore, every vertex of the tessellation coincides with some cell apices (cellular 0-faces) and every tessellation edge is a subset of some cell ridges (cellular 1-faces). So a vertex cannot lie in a facet- or ridge-interior and an edge-interior cannot lie in a facet-interior. We further note that a plate-side (an entity which is not always a face of a cell) is always contained in a ridge (which is), so also a vertex cannot lie in a plate-side interior. So the lemma is now proven.

Non facet-to-facet tessellations are not cell complexes and that perhaps explains why their study has been neglected.

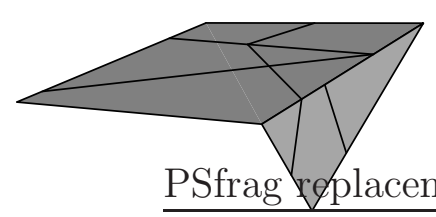

(a)

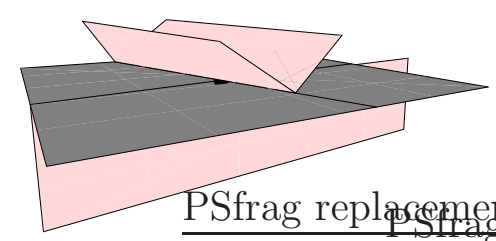

(b)

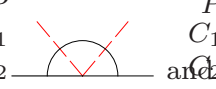

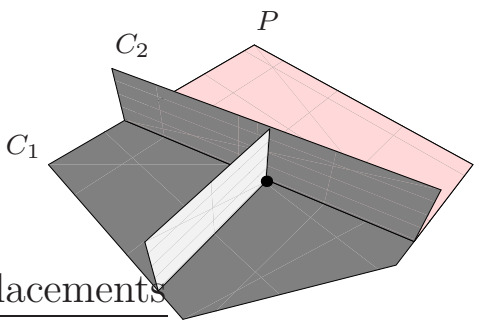

(c)

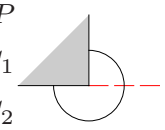

Figure 2: The schematic diagrams below the main figures are helpful in lemmas involving $\psi$ and $\tau$, as will be explained in Section 7 for the reader who does not guess their meaning immediately. The main figures are as follows. (a) Two facets of a cell $z$ are shown, with their interior structure due to neighbouring cells of $z$ (not shown). The dark-shaded facet (comprising six plates) has two hemi-vertices in its interior and seven $\pi$-edges. The lighter facet (comprising three plates) demonstrates that $\pi$-edges can exist on a facet without there being hemi-vertices. A ridge is also illustrated, with three interior vertices, two of which lie in the interior of a plate-side (whose plate is one of the nine shown). (b) A vertex adjacent to three plate-side-interiors and five ridge-interiors, but no facet-interior. (c) A common type of hemi-vertex, with $P$ and $C_{1}$ being coplanar. The vertex is in the interior of one plate-side (that of the plate $P$ ) and one ridge (where $P$ and $C_{2}$ meet). The labels $C_{1}$ and $C_{2}$ are bookcovers, a concept explained later.

The general theory and our findings: Section 6 shows how the interior parameters are constrained for those non facet-to-facet tessellations conforming with (3). There are non facetto-facet cases which do not conform to (3), because the upper bound for $\mu_{E P}$ no longer holds and the lower bound for $\mu_{P V}$ becomes more complicated. Sections 7 and 8 also deals with these 
cases; indeed one general theory presented in these sections covers all the non facet-to-facet cases.

It is convenient now to inform the reader of the major findings of our general theory.

THEOREM 1: MAIN RESUlT.

Facet-to-facet case: For tessellations which are facet-to-facet, the constraints on the three cyclic parameters $\mu_{V E}, \mu_{E P}$ and $\mu_{P V}$ are as follows:

$$
4 \leq \mu_{V E} ; \quad 3 \leq \mu_{E P} \leq 6\left(1-\frac{2}{\mu_{V E}}\right)<6 ; \quad 3 \leq \mu_{P V}<\frac{\mu_{V E} \mu_{E P}}{\mu_{V E}-2} \leq 6 .
$$

In this facet-to-facet case, the interior parameters $\xi, \kappa, \psi$ and $\tau$ all equal zero.

Non facet-to-facet case: A tessellation is not facet-to-facet if and only if $\xi>0$.

Some tessellations which are not facet-to-facet, have cyclic parameters which conform to (5), but many do not. The precise constraints in this case are as follows:

$$
4 \leq \mu_{V E} ; \quad 3 \leq \mu_{E P} ;\left\{\begin{array}{rlrl}
3 & \leq \mu_{P V}<\frac{\mu_{V E} \mu_{E P}}{\mu_{V E}-2}<6 & & \text { if } \mu_{E P}<6\left(1-\frac{2}{\mu_{V E}}\right) \\
\frac{\mu_{V E} \mu_{E P}}{2\left(\mu_{V E}-2\right)}<\mu_{P V}<\frac{\mu_{V E} \mu_{E P}}{\mu_{V E}-2} & \text { if } \mu_{E P} \geq 6\left(1-\frac{2}{\mu_{V E}}\right) .
\end{array}\right.
$$

The lower bound for $\mu_{P V}$ in the second case is $>3$ if and only if $\mu_{E P}>6\left(1-2 / \mu_{V E}\right)$ whilst the upper bound for $\mu_{P V}$ in this case is $>6$.

The permitted ranges for $\psi$ and $\tau$, given $\mu_{V E}, \mu_{E P}$ and $\mu_{P V}$, are as follows.

$$
\begin{gathered}
0 \leq \psi \leq\left\{\begin{array}{lc}
\mu_{V E}-2+\frac{\mu_{V E} \mu_{E P}}{2}\left(1-\frac{4}{\mu_{P V}}\right) & \text { if } \mu_{P V} \leq \frac{2 \mu_{V E} \mu_{E P}}{3 \mu_{V E}-8} \\
\frac{\mu_{V E}}{4}+\frac{\mu_{V E} \mu_{E P}}{2}\left(1-\frac{3}{\mu_{P V}}\right) & \text { if } \mu_{P V} \geq \frac{2 \mu_{V E} \mu_{E P}}{3 \mu_{V E}-8} .
\end{array}\right. \\
\max \left[0, \psi-\frac{\mu_{V E}}{2}, \frac{\psi}{2}+\frac{\mu_{V E}}{4}\left(\mu_{E P}-6\left(1-\frac{2}{\mu_{V E}}\right)\right)\right] \leq \tau \leq \min \left[\psi, \frac{1}{2} \mu_{V E} \mu_{E P}\left(1-\frac{3}{\mu_{P V}}\right)\right] .
\end{gathered}
$$

The permitted range for $\kappa$ and $\xi$, given $\mu_{V E}, \mu_{E P}, \mu_{P V}, \psi$ and $\tau$, is given by

$$
\begin{gathered}
0 \leq \kappa \leq \min \left[1, \mu_{V E}-2+\frac{1}{2} \mu_{V E} \mu_{E P}\left(1-\frac{4}{\mu_{P V}}\right)-\psi\right] \quad \text { and } \\
\max \left[\frac{2(\psi-\tau)+3 \kappa}{\mu_{V E}}, \frac{4 \psi+6 \kappa}{\mu_{V E}}-2 \mu_{E P}\left(1-\frac{3}{\mu_{P V}}\right)\right] \leq \xi \leq \min \left[1,3-\frac{\mu_{E P}}{2}+\frac{\psi-6+3 \kappa}{\mu_{V E}}\right],
\end{gathered}
$$

supplemented by $\xi>0$.

Our strategy: It is not easy to present graphically the shape of a seven-dimensional constraint space. Our method to give some visual experience of the domain displays $\mu_{E P}, \mu_{P V}$ and the four interior parameters in three two-dimensional plots: the $\mu_{E P}$ versus $\mu_{P V}$ permitted region given $\mu_{V E}$; the $\tau$ versus $\psi$ permitted region, once the three cyclic parameters are given; the permitted $\kappa$ versus $\xi$ region once the other five parameters have been decided. This follows the way Theorem 1 has been organised. A plot based on our Example 10(e), is presented as Figure 3 . 


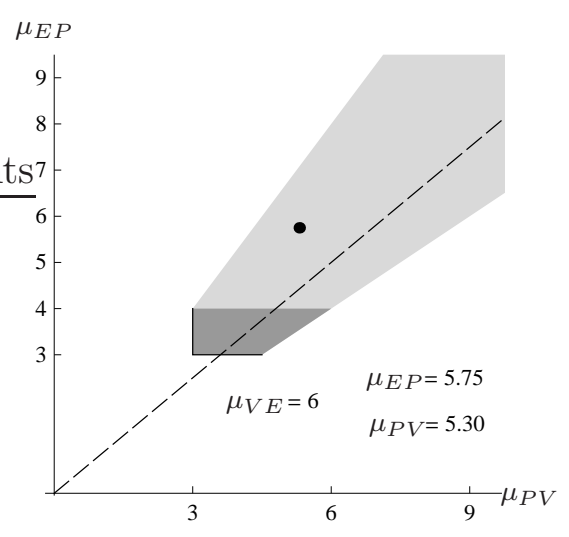

(a)

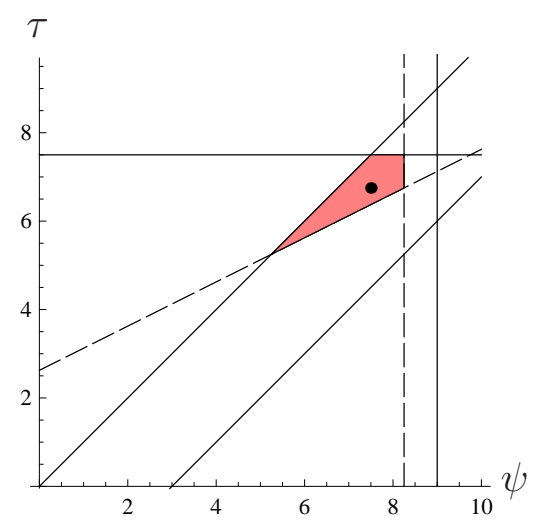

(b)

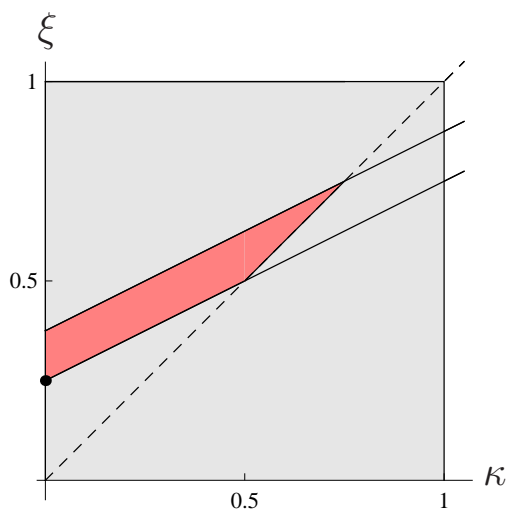

(c)

Figure 3: (a) The permitted range for $\left(\mu_{P V}, \mu_{E P}\right)$ if $\mu_{V E}=6$. The dark shading, bounded above by the line $\mu_{E P}=$ $6\left(1-2 / \mu_{V E}\right)$, is the range for facet-to-facet tessellations. It is 'open' on its right boundary, but 'closed' on the other boundaries. Some tessellations which are not facet-to-facet have $\left(\mu_{P V}, \mu_{E P}\right)$ in the dark zone but, for others, the point lies in the light grey zone which is an open set, unbounded above. The dashed line divides the region into two parts based on the inequalities in (7). The black dots in the three diagrams correspond to the actual values of Example 10(e), described later. (b) Given $\mu_{V E}=6$ and the $\left(\mu_{P V}, \mu_{E P}\right)$ dot in (a), the dark region shows the permitted region for $(\psi, \tau)$. The constraints, which are straight lines, come from (7] and [8). (c) The point $(\kappa, \xi)$ lies in the light grey square $[0,1] \times(0,1]$, but further contraints given in Theorem 1 lead to a smaller region, shown in darker shading. This plot assumes that the values of the other five parameters have been given.

For the $\tau$ versus $\psi$ region, the boundaries are determined firstly by the cyclic parameters and secondly by the requirement that the region does not make the $\xi$ versus $\kappa$ region null. Likewise, the region $\mu_{E P}$ versus $\mu_{P V}$ depends firstly on $\mu_{V E}$ and secondly on the need to have non-null regions for the interior parameters. As for $\mu_{V E}$ itself, we also investigate if there is any value it can take in its range $[4, \infty)$ that renders null the regions for the other six parameters. The reader will see these strategies used in the arguments that lead to Theorem 1.

Note that whilst we prove Theorem 1, space does not permit us to prove all of the facts quoted in our example base. Separate papers or supportive technical notes ([3], [10]) provide some proofs in the most substantial examples; they also give further extension and graphical demonstrations of these examples.

REMARK 1: Before commencing the agenda set out above, we note some very basic information. In a tessellation with convex cells, the cells must be polyhedra. Also, every vertex of the tessellation must have at least four emanating edges and every edge at least three emanating plates; otherwise, non-convex cells would exist. So, $\mu_{V E} \geq 4$ and $\mu_{E P} \geq 3$. Plates are polygons and therefore have no fewer than three corners, so we can state that $\mu_{P V} \geq 3$. Thus the left-hand bound of the inequalities in (5) and, in part, (6) are proved trivially.

REMARK 2: Cells are convex polyhedra, so we can use some simple inequalities that apply to polyhedra. For any convex polyhedron with $f_{k}$ faces of dimension $k$, and therefore for any cell of the spatial tessellation,

$$
\begin{aligned}
& 3 f_{0} \leq 2 f_{1} \text { and } \\
& 3 f_{2} \leq 2 f_{1}
\end{aligned}
$$




\section{Examples}

We commence with well-known examples of random stationary tessellations in $\mathbb{R}^{3}$.

- Example 1: The Voronoi tessellation based on seeds from a stationary Poisson point process. Here $\left(\mu_{V E}, \mu_{E P}\right)=(4,3)$ and $\mu_{P V}=144 \pi^{2} /\left(24 \pi^{2}+35\right) \approx 5.2$. This is the best known facet-to-facet tessellation (see [11]).

- Example 2: The Delaunay tessellation, with similar seeds (also see [1]). Here we have $\left(\mu_{V E}, \mu_{E P}\right)=\left(2+48 \pi^{2} / 35,144 \pi^{2} /\left(24 \pi^{2}+35\right)\right) \approx(15.5,5.2)$. All plates are triangular and all cells are tetrahedra (so, being a facet-to-facet tessellation, $\mu_{P V}=3$ whilst $\mu_{Z V}=$ $\mu_{Z P}=4$ and $\left.\mu_{Z E}=6\right)$.

- Example 3: The tessellation formed by random planes, no four of which meet at a point $([7])$. Note that $\left(\mu_{V E}, \mu_{E P}\right)=(6,4)$. Also $\mu_{P V}=4$ if the plane process is Poisson.

- Example 4: The STIT tessellation in $\mathbb{R}^{3}$ (see 9]). This is our first example that is not facet-to-facet. It is known from [9] that $\mu_{V E}=4, \mu_{E P}=3$ and $\mu_{P V}=\frac{36}{7}$ and from [15] (also [14]) that $\xi=1, \kappa=\frac{2}{3}, \psi=2$ and $\tau=\frac{4}{3}$.

- Example 5: Cubes packed in a lattice. Note that all tilings based on a repeating subunit can be converted to a random stationary tessellation by locating the origin uniformly distributed within the sub-unit. The cyclic parameters are the same as in Example 3.

Each of the Examples 1-5 provide dots for the plot of Figure1, they are annotated by 'example number'. Also on the figure are dots which belong to a class of models. For example, we shall introduce our sixth 'model' as a class.

- Example(s) 6: Congruent prisms arranged in columns. The triangular prism model, model 6a, is depicted in Figure 4(b) and analysed in [15]. All cells are congruent triangular prisms with parallel longitudinal axes and front facets which are not aligned with each other. The prisms are arranged in columns. Similarly packed structures with all cells congruent and arranged in columns can be achieved with quadrilateral, pentagonal or hexagonal front facets (models $6 \mathrm{~b}, 6 \mathrm{c}$ and $6 \mathrm{~d}$ respectively). In each case, we confine our attention to models where the planar tessellation, on a plane cut orthogonal to the column axis, is side-to-side and comprising, of course, congruent polygons for cells. All of these models have $\mu_{V E}=4, \xi=\frac{1}{2}$ and $\kappa=0$, but the other parameters differ.

(a) triangular prisms: $\mu_{E P}=\frac{9}{2}, \mu_{P V}=\frac{27}{4}, \psi=5$ and $\tau=4$.

(b) quadrilateral prisms: $\mu_{E P}=\frac{7}{2}, \mu_{P V}=\frac{28}{5}, \psi=3$ and $\tau=2$.

(c) pentagonal prisms (based on the so called Cairo side-to-side tessellation of the plane with congruent pentagons, see Wells [17], p.23): $\mu_{E P}=\frac{16}{5}, \mu_{P V}=\frac{16}{3}, \psi=\frac{12}{5}$ and $\tau=\frac{7}{5}$.

(d) hexagonal prisms: $\mu_{E P}=3, \mu_{P V}=\frac{36}{7}, \psi=2$ and $\tau=1$.

These models belongs to a larger class of "column tessellations" discussed in Section 5.

Then follow a number of examples constructed by adding dividing planes to some or all of the cells in Examples 1-6. 


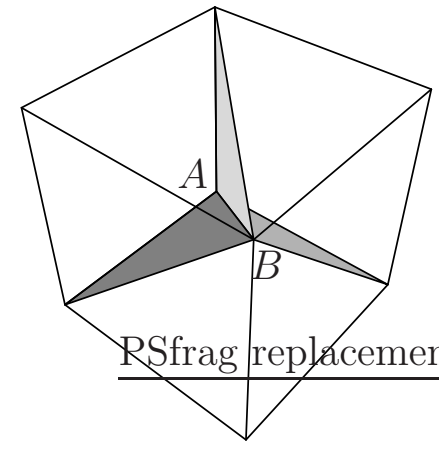

(a)

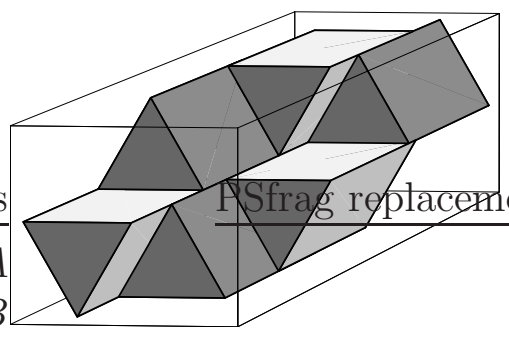

(b)

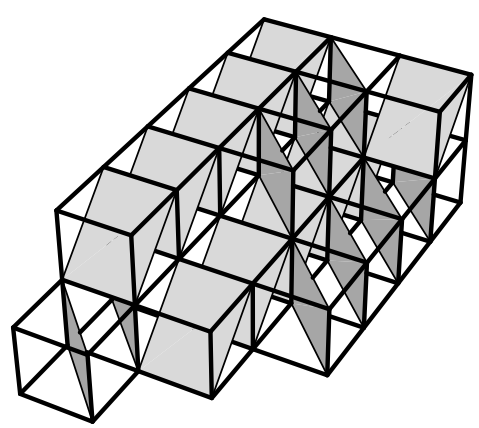

(c)

Figure 4: An illustration of (a) a partition of the cube used in Examples 7 and 16, (b) Example 6a (with the schematic, which applies to the visually most left vertex of the most right prism, defined in Section 7) and (c) Example 15.

- Example 7: A cube can be divided into three congruent pyramids. This is achieved with three congruent triangular plates which meet on one diagonal $A B$ of the cube, as shown in Figure 4(a). Of the six facets of the original cube, three remain as undivided squares (see those facets adjacent to $A$ ) whilst three are divided by a diagonal chord (those adjacent to $B$ ). The diagonal chosen for each cube could be randomised in some way, but here we choose a non-random way that produces a facet-to-facet tessellation. Consider a larger cube containing eight of the original cubes. Now, starting from the centre of the large cube draw diagonals into each of the eight smaller cubes. Place three triangular plates in each cell meeting on this diagonal, choosing the method which puts the end $B$ (the corner where these triangles have their smallest angle) in the centre of the large cube. So, no parts of the 16 added plates are visible from outside the large eight-celled cube because only the faces adjacent to $A$ are on the outside surface of this large cube. Then we tessellate $\mathbb{R}^{3}$ as a cubic lattice using the large cube. Here $\left(\mu_{V E}, \mu_{E P}\right)=(8,4)$ and $\mu_{P V}=\frac{16}{5}$.

- Example 8: Each cell of a Delaunay tessellation is a tetrahedron. We independently divide each cell into two tetrahedra by joining one of the cell's ridges, randomly chosen from the six it has, to a uniformly random point on the opposite ridge. The Delaunay is facet-to-facet, but the Divided Delaunay is not. This model is analysed in full in [15] where it is shown that $\mu_{V E}=\frac{14\left(5+16 \pi^{2}\right)}{35+32 \pi^{2}} \approx 6.50, \mu_{E P}=\frac{72 \pi^{2}\left(175+176 \pi^{2}\right)}{7\left(5+16 \pi^{2}\right)\left(35+24 \pi^{2}\right)} \approx 4.38, \mu_{P V}=$ $\frac{9\left(175+176 \pi^{2}\right)}{16\left(35+24 \pi^{2}\right)} \approx 3.96, \xi=\frac{64 \pi^{2}}{7\left(5+16 \pi^{2}\right)} \approx 0.55, \kappa=0, \psi=\frac{\left.8 \pi^{2}\left(35+528 \pi^{2}\right)\right)}{\left(35+24 \pi^{2}\right)\left(35+32 \pi^{2}\right)} \approx 4.34$ and $\tau=$ $\frac{32 \pi^{2}\left(102 \pi^{2}-35\right)}{\left(35+24 \pi^{2}\right)\left(35+32 \pi^{2}\right)} \approx 3.22$.

The construction in our next class of models starts with a planar tessellation, so firstly a few remarks on notation will be apposite. The notations $\mu_{X Y}$ and $\lambda_{X}$ are also used in the planar case and only one adjacency, $\mu_{V E}$, and one intensity, $\lambda_{V}$, are required to express all others. Only one 'interior' parameter, namely $\phi$, is required ([1],[2]).

- $\phi:=$ the proportion of vertices which are contained in the interior of some cell-side. Note that $\phi=\mu_{V \stackrel{\circ}{Z}_{1}}$. 
These are called $\pi$-vertices because one angle between consecutive edges emanating from the vertex is $\pi$. Planar tessellations are side-to-side if $\phi=0$, or not if $\phi>0$. Naturally $0 \leq \phi \leq 1$ and, as shown in [15], $3 \leq \mu_{V E} \leq 6-2 \phi$. ( This is a smaller constraint domain for $\mu_{V E}$ than the region $3 \leq \mu_{V E} \leq 6$ given in [4]; in that study, $\phi$ was not used.)

- Example(s) 9; the stratum models: Given any stationary tessellation $\mathcal{Y}^{\prime}$ in the plane, we can construct a tessellation of $\mathbb{R}^{3}$ by firstly making each cell of $\mathcal{Y}^{\prime}$ into a prism of depth 1 unit by adding plates orthogonal to the plane in which $\mathcal{Y}^{\prime}$ lies. This forms a stratum of depth 1 . We then place further copies of the stratum next to the first (without any offset of $\mathcal{Y}^{\prime}$ ) and so on until $\mathbb{R}^{3}$ is filled. Mecke introduced this structure in [6], together with the name stratum. Given a $\left(\mu_{V E}^{\prime}, \phi\right)$-planar tessellation, we can write the seven parameters $\left(\mu_{V E}, \mu_{E P}, \ldots\right)$ for the stratum model as functions of $\mu_{V E}^{\prime}$ and $\phi$.

$$
\begin{array}{ll}
\mu_{V E}=\mu_{V E}^{\prime}+2 & \xi=\frac{2 \phi}{\mu_{V E}^{\prime}+2} \\
\mu_{E P}=\frac{6 \mu_{V E}^{\prime}}{\mu_{V E}^{\prime}+2} & \psi=2 \phi \\
\mu_{P V}=\frac{3 \mu_{V E}^{\prime}}{\mu_{V E}^{\prime}-1} & \kappa=0 .
\end{array}
$$

Note that the three-dimensional tessellation is facet-to-facet if and only if the planar tessellation is side-to-side (which is equivalent to $\phi=0$ ).

The proof of these identities is deferred to a supportive paper [10]. We note here that all stratum models lie on the curve $\mu_{E P}=6\left(1-2 / \mu_{V E}\right)$ and with $5 \leq \mu_{V E} \leq 8-\psi$. Dots annotated $9 a-9 d$ on Figure 1 correspondent to (a)-(d) below.

(a) Stratum constructed from a Voronoi tessellation in the plane, so $\left(\mu_{V E}, \mu_{E P}\right)=\left(5, \frac{18}{5}\right)$ and $\mu_{P V}=\frac{9}{2}$.

(b) Stratum constructed from the planar Delaunay tessellation. Thus $\left(\mu_{V E}, \mu_{E P}\right)=$ $\left(8, \frac{9}{2}\right)$ and $\mu_{P V}=\frac{18}{5}$.

(c) Stratum constructed from the superposition of planar Voronoi tessellation and its dual Delaunay tessellations (see [15], Figure 1(a), where it is shown that $\mu_{V E}^{\prime}=4$ and $\phi=0)$. So $\left(\mu_{V E}, \mu_{E P}\right)=(6,4)$ and $\mu_{P V}=4$.

(d) Stratum constructed from the planar STIT tessellation. Cyclic parameters are the same as for $9 a$; also $\xi=\frac{2}{5}, \psi=2, \tau=1$ and $\kappa=0$.

Dots $9 a$ and $9 b$ in Figure 1 are the bounding cases.

- Example(s) 10; the central-point models: In this class of models, we commence with any stationary tessellation of $\mathbb{R}^{3}$. In the interior of some cell, a point $Q$ is placed. Then, from every apex of that cell, a line segment is drawn to $Q$. Also, from every ridge of the cell a triangular plate is constructed with base being the ridge and $Q$ the opposite corner. Repeat this for all cells. Thus, every cell is itself partitioned into many pyramids, as many as there are facets of the original cell. 
The parameters are as follows.

$$
\begin{array}{rlrl}
\mu_{V E} & =\frac{2 \mu_{P V}^{\prime}\left(\mu_{V E}^{\prime}-4-\mu_{V E}^{\prime} \mu_{E P}^{\prime}+2 \kappa^{\prime}+2 \psi^{\prime}\right)}{\mu_{P V}^{\prime}\left(\mu_{V E}^{\prime}-4\right)-\mu_{V E}^{\prime} \mu_{E P}^{\prime}} & \xi & =\frac{\mu_{V E}^{\prime} \xi^{\prime}}{\mu_{V E}^{\prime}\left(\mu_{E P}^{\prime}-1\right)+4-2 \kappa^{\prime}-2 \psi^{\prime}} \\
\mu_{E P}=\frac{4 \mu_{V E}^{\prime} \mu_{E P}^{\prime}-3 \mu_{V E}^{\prime} \xi^{\prime}-4 \psi^{\prime}}{4-\mu_{V E}^{\prime}+\mu_{V E}^{\prime} \mu_{E P}^{\prime}-2 \kappa^{\prime}-2 \psi^{\prime}} & \kappa & =\frac{2 \mu_{P V}^{\prime} \kappa^{\prime}}{\mu_{V E}^{\prime} \mu_{E P}^{\prime}-\mu_{P V}^{\prime}\left(\mu_{V E}^{\prime}-4\right)} \\
\mu_{P V}=\frac{\mu_{P V}^{\prime}\left(4 \mu_{V E}^{\prime} \mu_{E P}^{\prime}-3 \mu_{V E}^{\prime} \xi^{\prime}-4 \psi^{\prime}\right)}{\mu_{V E}^{\prime} \mu_{E P}^{\prime}\left(\mu_{P V}^{\prime}+1\right)-\mu_{P V}^{\prime}\left(\mu_{V E}^{\prime} \xi^{\prime}+2 \psi^{\prime}\right)} & \psi & =\frac{4 \mu_{P V}^{\prime} \psi^{\prime}}{\mu_{V E}^{\prime} \mu_{E P}^{\prime}-\mu_{P V}^{\prime}\left(\mu_{V E}^{\prime}-4\right)} \\
\tau & =\frac{2 \mu_{P V}^{\prime}\left(\tau^{\prime}+\psi^{\prime}\right)}{\mu_{V E}^{\prime} \mu_{E P}^{\prime}-\mu_{P V}^{\prime}\left(\mu_{V E}^{\prime}-4\right)} .
\end{array}
$$

The dots marked $10 a, 10 b, \ldots$ in Figure 1 are constructed from the following models:

(a) a Voronoi tessellation of $\mathbb{R}^{3}$ and yielding $\left(\mu_{V E}, \mu_{E P}\right)=\left(\frac{288 \pi^{2}}{35+24 \pi^{2}}, 4\right)$ and $\mu_{P V}=$ $\frac{576 \pi^{2}}{7\left(5+24 \pi^{2}\right)}$

(b) a Delaunay tessellation, yielding $\left(\mu_{V E}, \mu_{E P}\right)=\left(\frac{10\left(7+24 \pi^{2}\right)}{35+24 \pi^{2}}, \frac{576 \pi^{2}}{7\left(5+24 \pi^{2}\right)}\right)$ and $\mu_{P V}=3$;

(c) the STIT tessellation, with $\left(\mu_{V E}, \mu_{E P}\right)=\left(\frac{40}{7}, \frac{21}{5}\right), \mu_{P V}=\frac{84}{19}, \xi=\frac{3}{5}, \kappa=\frac{4}{7}, \psi=\frac{24}{7}$ and $\tau=\frac{20}{7}$

(d) the cubic lattice tessellation, with $\left(\mu_{V E}, \mu_{E P}\right)=\left(11, \frac{48}{11}\right)$ and $\mu_{P V}=\frac{16}{5}$;

(e) the triangular prism tessellation, defined in Example $6(\mathrm{a})$ and yielding $\left(\mu_{V E}, \mu_{E P}\right)=$ $\left(6, \frac{23}{4}\right), \mu_{P V}=\frac{69}{13}, \xi=\frac{1}{4}, \kappa=0, \psi=\frac{15}{2}$ and $\tau=\frac{27}{4} ;$

(f) - (i) further iterations of $6(\mathrm{a})$.

These iterations of Example 6(a) are connected by line segments in Figure 1 . Note that, because iteration decreases $\xi$, the iterates have $\left(\mu_{V E}, \mu_{E P}\right)$ points which are moving toward the region $\mu_{E P} \leq 6\left(1-2 / \mu_{V E}\right)$ where tessellations with $\xi=0$ can exist. In the limit, these iterations of $\left(\mu_{V E}, \mu_{E P}\right)$ reach $\left(8, \frac{9}{2}\right)$ where Example $9(\mathrm{~b})$ is positioned.

Note that the constructed tessellations in Examples 10 are facet-to-facet if and only if the starting tessellation is also facet-to-facet.

\section{Constraints in the facet-to-facet case}

In this section we assume that the tessellation is facet-to-facet. Consider again the inequalities (9) and (10). They apply to every cell of the tessellation. So, $\mu_{Z V}$ equals the average of $f_{0}$ over all cells. Likewise, $\mu_{Z E}$ and $\mu_{Z P}$ are the averages of $f_{1}$ and $f_{2}$ respectively. So (9) leads to

$$
3 \mu_{Z V} \leq 2 \mu_{Z E}
$$

which in turn (from the entries in Table 1) is equivalent to

$$
\frac{\mu_{P V} f(2)}{f\left(\mu_{P V}\right)} \leq \frac{\mu_{V E} \mu_{E P} \mu_{P V}}{f\left(\mu_{P V}\right)}
$$

or to

$$
\mu_{E P} \leq 6\left(1-\frac{2}{\mu_{V E}}\right)
$$




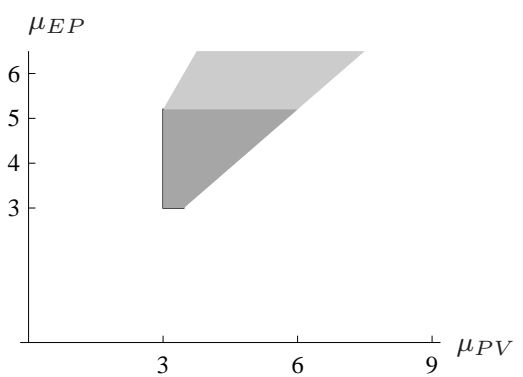

(a) $\mu_{V E}=15$

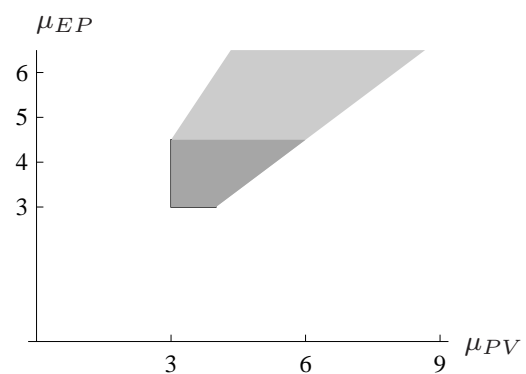

(b) $\mu_{V E}=8$

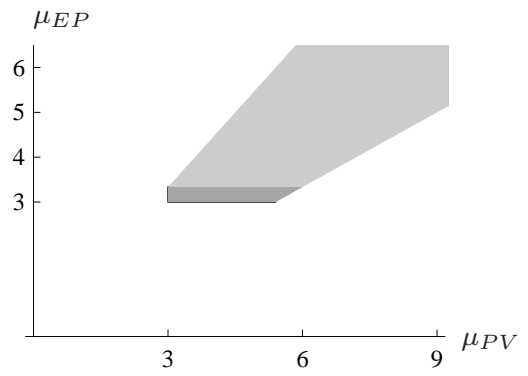

(c) $\mu_{V E}=\frac{9}{2}$

Figure 5: The darkly shaded region is the range of $\left(\mu_{P V}, \mu_{E P}\right)$ for various $\mu_{V E}$ when the tessellation is facet-to-facet. Note that the upper and lower boundaries of the dark zone coincide when $\mu_{V E}=4$. We shall see later, in Section 6 , the theory behind the light grey region. This region, an open set bounded on the left by the sloping line $\mu_{E P}=2\left(1-2 / \mu_{V E}\right) \mu_{P V}$, is the range for non facet-to-facet tessellations which have $\tau>0$. The sloping line which bounds both shaded regions on the right is $\mu_{E P}=\left(1-2 / \mu_{V E}\right) \mu_{P V}$. Tessellations which are not facet-to-facet and have $\tau=0$ are also in the darkly-shaded region (see Remark 9). The non facet-to-facet theory is described later in the paper.

This becomes a fundamental inequality for facet-to-facet tessellations. The equality in (12) holds if and only if all cells are simple polyhedra - which means that each apex of the polyhedron must have three ridges of the polyhedron emanating from it. The other inequality, (10), is less fruitful as it is equivalent to something we know already, namely $\mu_{P V} \geq 3$.

Turning now to the rest of Table 1 , we can systematically write inequalities attached to many of the entries - for example, $\mu_{Z V} \geq 4, \mu_{Z E} \geq 6, \mu_{Z P} \geq 4$ and $f\left(\mu_{P V}\right)>0$. The first three of these provide lower bounds for $\mu_{P V}$, but all of these are weaker than $\mu_{P V} \geq 3$ when (12) holds - as it does when the tessellation is facet-to-facet. However, $\lambda_{Z}>0 \Longleftrightarrow f\left(\mu_{P V}\right)>0$ which is equivalent to

$$
\mu_{P V}<\frac{\mu_{V E} \mu_{E P}}{\mu_{V E}-2}
$$

this upper bound for $\mu_{P V}$ being $\leq 6$ in the facet-to-facet case because of (12). We have thus completed the proof of (5). The darkly shaded regions of Figure 5 show the permitted range for $\left(\mu_{P V}, \mu_{E P}\right)$ for various $\mu_{V E}$, illustrating the inequalities in (5).

\section{Facet-to-facet examples with large $\mu_{V E}$ or large $\mu_{Z V}$}

Figure 6] shows how some parameters in a stationary tessellation of $\mathbb{R}^{3}$ can be arbitrarily large. This is in contrast with the situation for planar tessellations. Both 6)(a) and 6(b) are based on the cubic lattice tessellation, with structure added in the interior of each cube.

- Example 11; model with unbounded $\mu_{V E}$ : In Figure 6(a), a vertical line joins the centres of the top and bottom facets of the cube. Called these centres $T$ and $B$. This line $T B$ is further divided into $(k+1)$ edges by the addition of $k$ equally-spaced vertices $V_{1}, V_{2}, \ldots, V_{k}$. The case $k=1$ is shown in Figure 6(a). Around the boundary of the bottom facet are placed $4 n$ vertices in addition to those at the face's corners, $n$ in the interior of each side of the square facet. Figure 6(a) shows the case $n=2$. Similarly, the top facet has these added vertices on its boundary. From each vertex $V_{j}, j=1,2, \ldots, k$, and $T$ and $B$ too, edges are drawn to the $4(n+1)$ vertices on the boundary of the bottom facet (as shown). Edges also join $T$ with the $4(n+1)$ vertices on the boundary of the top facet. Finally $T$ and $B$ are joined, creating many edges because the joining line-segment 


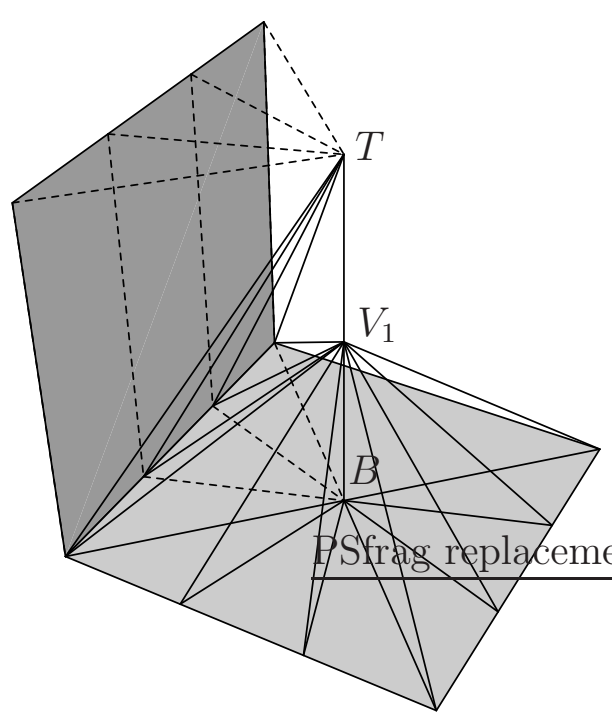

(a)

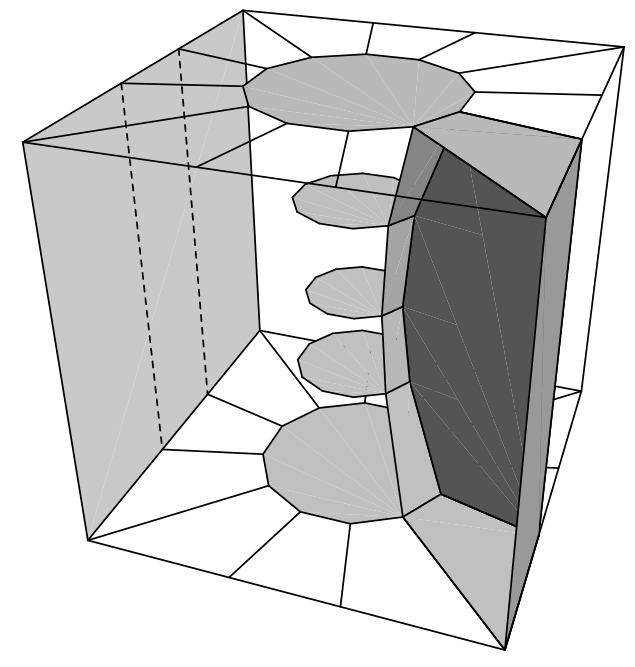

(b)

Figure 6: (a) Model with unbounded $\mu_{V E}$; (b) model with unbounded $\mu_{Z V}$.

passes though $V_{j}, j=1,2, \ldots, k$. Plates are now formed in the obvious places seen in the drawing. A facet-to-facet tessellation results.

It can be shown that:

$$
\mu_{V E}=\frac{2(12+5 k+12 n+4 k n)}{2+k+2 n} ; \quad \mu_{V P}=\frac{8(7+3 k)(1+n)}{2+k+2 n} ; \quad \mu_{V Z}=\frac{4(9+4 k)(1+n)}{2+k+2 n} .
$$

So, $\mu_{V E}$ can be made arbitrarily large (and this reflects in the other mean adjacencies $\mu_{V P}$ and $\mu_{V Z}$ associated with the typical vertex). All other adjacencies are bounded as $k$ and/or $n$ becomes large. For example:

$$
\mu_{E P}=\frac{8(7+3 k)(1+n)}{12+5 k+12 n+4 k n} ; \quad \quad \mu_{P V}=\frac{4(7+3 k)}{9+4 k} .
$$

Some points for small $n$ and $k$ appear on the plot of Figure1, annotated $(k, n)=(2,0)$ as '11a', $(k, n)=(0,0)$ as ' $11 \mathrm{~b}$ ' and $(k, n)=(2,1)$ as '11c'. For larger $k$ and $n$, the points lie off the graph to the right, but always below the curve (approaching the curve as $k \rightarrow \infty$ ).

- Example 12; model with unbounded $\mu_{Z V}$ : In Figure 6(b), there are also $4(n+1)$ vertices on the boundary of both the bottom and top facets of the cube. There is a central core made up of $k+1$ prisms, each bounded above and below by a $4(n+1)$-sided polygonal plate. Other cells are constructed outside the central core as per the illustration (which uses $n=2$ and $k=3$ ). The tessellation is facet-to-facet, with:

$$
\mu_{Z V}=\frac{8(5+2 k)(1+n)}{5+k+4 n} ; \quad \mu_{Z E}=\frac{12(5+2 k)(1+n)}{5+k+4 n} ; \quad \mu_{Z P}=\frac{2(15+5 k+14 n+4 k n)}{5+k+4 n} .
$$

We note that these adjacencies can be made arbitrarily large. Our cyclic trio are, however, bounded above.

$$
\begin{aligned}
& \mu_{V E}=\frac{2(15+8 k+16 n+8 k n)}{5+4 k+6 n+4 k n} ; \\
& \mu_{E P}=\frac{12(5+2 k)(1+n)}{15+8 k+16 n+8 k n} ; \\
& \mu_{P V}=\frac{12(5+2 k)(1+n)}{15+5 k+14 n+4 k n} .
\end{aligned}
$$


These formulae lead to a datum for Figure 1, for the case $n=k=3$. All such data for this example would lie on the fundamental curve, with $4 \leq \mu_{V E} \leq 6$.

The examples above in this section show rather extreme behaviour, demonstrating the unbounded character of $\mu_{V E}$ and $\mu_{Z V}$ - something already noted for facet-to-facet tilings by Ziegler at a 2002 conference (reported in [18]).

Example 11 is especially useful to our Figure 1, as it shows that valid tessellations exist far to the right of the diagram, below the curve. It is also useful in a rather esoteric way, in mixtures of models.

\section{Tessellations which are mixtures}

Formally, a random tessellation is a mapping from a probability space $(\Omega, \mathcal{T}, \mathrm{P})$ into the space of all tessellations of the holding space, here $\mathbb{R}^{3}$. The probability measure $P$ can be of the form $\alpha \mathrm{P}_{1}+(1-\alpha) \mathrm{P}_{2}$ where $0 \leq \alpha \leq 1, \mathrm{P}_{1}$ and $\mathrm{P}_{2}$ being probability measures. In practical terms, this means that with probability $\alpha$ one tessellation model is realised and with probability $(1-\alpha)$ it is another model. As $\alpha$ varies through the interval [0,1], a curve is traced between the points of Figure 1 which represent $P_{1}$ and $P_{2}$ and all mixtures of these lie on the curve.

- Example(s) 13; Two mixture tessellations and some mixture curves. For example, the dashed curves in Figure 1 show the mixture curves of 8 and 7, $10 e$ and 2 and $6 a$ with model 11 (having $k=n=100$ and therefore its $\left(\mu_{V E}, \mu_{E P}\right)$ point located far to the right of the region shown and a very small distance below the solid curve). Both the dots marked $13 a$ and $13 b$ are mixtures of $2\left(\right.$ as $\left.\mathrm{P}_{1}\right)$ and $10 e\left(\right.$ as $\left.\mathrm{P}_{2}\right)$ with $\alpha=0.8$. In $13 b$ the vertex intensities of the two pure models are equal, whilst in $13 a$ the vertex intensity of $10 e$ is twice that of 2 .

Theory of mixture curves: When we mix two tessellations of $\mathbb{R}^{3}$, we can calculate the properties of the mixture from the following two identities (using suitable choices for the object classes $X$ and $Y$ ). Using a superscript $[j]$ (note the square brackets) to indicate that $\mathrm{P}_{j}$ is operative, we can write the following:

$$
\begin{aligned}
\lambda_{X} & =\alpha \lambda_{X}^{[1]}+(1-\alpha) \lambda_{X}^{[2]} \\
\mu_{X Y} & =\frac{\lambda_{X}^{[1]} \alpha \mu_{X Y}^{[1]}+\lambda_{X}^{[2]}(1-\alpha) \mu_{X Y}^{[2]}}{\alpha \lambda_{X}^{[1]}+(1-\alpha) \lambda_{X}^{[2]}} .
\end{aligned}
$$

The data points in Figure 1 are $\left(\mu_{V E}, \mu_{E P}\right)$. If such a point is a mixture, we can write:

$$
\begin{aligned}
& \mu_{V E}=\frac{\lambda_{V}^{[1]} \alpha \mu_{V E}^{[1]}+\lambda_{V}^{[2]}(1-\alpha) \mu_{V E}^{[2]}}{\alpha \lambda_{V}^{[1]}+(1-\alpha) \lambda_{V}^{[2]}} ; \\
& \mu_{E P}=\frac{\lambda_{E}^{[1]} \alpha \mu_{E P}^{[1]}+\lambda_{E}^{[2]}(1-\alpha) \mu_{E P}^{[2]}}{\alpha \lambda_{E}^{[1]}+(1-\alpha) \lambda_{E}^{[2]}} .
\end{aligned}
$$

As $\alpha$ moves through the interval [0, 1], a continuous curve is traced from $\left(\mu_{V E}^{[2]}, \mu_{E P}^{[2]}\right)$ to $\left(\mu_{V E}^{[1]}, \mu_{E P}^{[1]}\right)$. Eliminating $\alpha$ gives the curve $\left(\mu_{E P}\right.$ versus $\left.\mu_{V E}\right)$ as

$$
\mu_{E P}=\frac{\mu_{E P}^{[1]} \mu_{V E}^{[1]}-\mu_{E P}^{[2]} \mu_{V E}^{[2]}}{\mu_{V E}^{[1]}-\mu_{V E}^{[2]}}-\frac{\left(\mu_{E P}^{[1]}-\mu_{E P}^{[2]}\right) \mu_{V E}^{[1]} \mu_{V E}^{[2]}}{\mu_{V E}\left(\mu_{V E}^{[1]}-\mu_{V E}^{[2]}\right)}
$$


when $\mu_{V E}^{[1]} \neq \mu_{V E}^{[2]}$. When $\mu_{V E}^{[1]}=\mu_{V E}^{[2]}$, the curve is a vertical line-segment joining the two points.

Note that the result in (14) is valid whether $\lambda_{V}^{[1]}=\lambda_{V}^{[2]}$ or not. Also note that, if both $\left(\mu_{V E}^{[1]}, \mu_{E P}^{[1]}\right)$ and $\left(\mu_{V E}^{[2]}, \mu_{E P}^{[2]}\right)$ lie on a curve of the form $\mu_{E P}=A-B / \mu_{V E}$, where $A$ and $B$ are constants, (14) lies on the same curve as shown by the following calculation.

$$
\begin{aligned}
\mu_{E P} & =\frac{\left(A-B / \mu_{V E}^{[1]}\right) \mu_{V E}^{[1]}-\left(A-B / \mu_{V E}^{[2]}\right) \mu_{V E}^{[2]}}{\mu_{V E}^{[1]}-\mu_{V E}^{[2]}}-\frac{\left[\left(A-B / \mu_{V E}^{[1]}\right)-\left(A-B / \mu_{V E}^{[2]}\right)\right] \mu_{V E}^{[1]} \mu_{V E}^{[2]}}{\mu_{V E}\left(\mu_{V E}^{[1]}-\mu_{V E}^{[2]}\right)} \\
& =\frac{A \mu_{V E}^{[1]}-A \mu_{V E}^{[2]}-B+B}{\mu_{V E}^{[1]}-\mu_{V E}^{[2]}}-\frac{B \mu_{V E}^{[1]}-B \mu_{V E}^{[2]}}{\mu_{V E}\left(\mu_{V E}^{[1]}-\mu_{V E}^{[2]}\right)} \\
& =A\left(1-\frac{B}{\mu_{V E}}\right) .
\end{aligned}
$$

Our fundamental curve is of this form, with $A=6$ and $B=12$, and is therefore closed under the mixture operation.

REMARK 3: Readers accustomed to non-random tilings may regard such mixtures of models as fraudulent. To them, the more interesting question is "what is the existence domain in Figure 1 using only non-mixture models?". We do not yet have an answer to this difficult question.

\section{Introductory theory for non facet-to-facet tessellations}

Proof of (6) for non facet-to-facet tessellations: The most obvious change from the facetto-facet theory that we dealt with in Section 3 is that (12), the upper bound on $\mu_{E P}$, is no longer valid. We can however retain (13), the upper bound on $\mu_{P V}$, because the argument for it remains sound in the non facet-to-facet case. It cannot now be argued, however, that this upper bound on $\mu_{P V}$ is $\leq 6$; the former argument had used (12).

We now address the lower bound for $\mu_{P V}$ in the non facet-to-facet case. To find this bound, we initially consider the entity $\mu_{Z V}$, the expected number of tessellation vertices adjacent to a typical cell. For $\mu_{Z V}$ to equal 4, it is necessary that all cells be tetrahedra - and for there to be no $\pi$-edges, because the vertices at the termini of any $\pi$-edges would raise $\mu_{Z V}$ above 4 . So

$\mu_{Z V}=4 \Longrightarrow \xi=0$. Therefore a non facet-to-facet tessellation has $\mu_{Z V}>4$; we use this fact to establish the bound (15) that follows. The entry for $\mu_{Z V}$ in Table 1 and the definition of $f$ in (1) are also used.

$$
\begin{aligned}
\text { non facet-to-facet } \Longrightarrow \mu_{Z V}>4 & \Longleftrightarrow \frac{\mu_{P V} f(2)}{f\left(\mu_{P V}\right)}>4 \\
& \Longleftrightarrow \frac{\mu_{P V}\left(\mu_{V E} \mu_{E P}-2\left(\mu_{V E}-2\right)\right)}{\mu_{V E} \mu_{E P}-\mu_{P V}\left(\mu_{V E}-2\right)}>4 \\
& \Longleftrightarrow \frac{\mu_{V E} \mu_{E P}}{2\left(\mu_{V E}-2\right)}<\mu_{P V} .
\end{aligned}
$$

This new lower bound for $\mu_{P V}$ is $>3$ if and only if $\mu_{E P}>6\left(1-2 / \mu_{V E}\right)$, so it plays no role in facet-to-facet tessellations that have $\mu_{Z V}>4$. The inequality $\mu_{P V} \geq 3$ remains operative for all tessellations, facet-to-facet or not, when $\mu_{E P}<6\left(1-2 / \mu_{V E}\right)$. Because

$$
\mu_{E P}=6\left(1-\frac{2}{\mu_{V E}}\right) \Longleftrightarrow \frac{\mu_{V E} \mu_{E P}}{2\left(\mu_{V E}-2\right)}=3
$$


(15) implies that $\mu_{P V}>3$ in the non facet-to-facet case when $\mu_{E P}=6\left(1-2 / \mu_{V E}\right.$ ) (though the inequality remains $\mu_{P V} \geq 3$ in the facet-to-facet case). Thus (6) in the main theorem is proven. An illustration of these inequalities is given in Figure 5 .

REMARK 4: When $\mu_{E P}>6\left(1-2 / \mu_{V E}\right)$, the lower bound $\frac{1}{2} \mu_{V E} \mu_{E P} /\left(\mu_{V E}-2\right)$ is also greater than lower bounds that can be similarly derived from $\mu_{Z E}>6$ and $\mu_{Z P}>4$.

A focus on $\mu_{E P}$ : We have seen above that our earlier proof of (12), which says that $\mu_{E P} \leq 6\left(1-2 / \mu_{V E}\right)$ in the facet-to-facet case, no longer holds. Figure 1, with its many black dots above the fundamental curve, goes further and demonstrates that $\mu_{E P}$ can be greater than $6\left(1-2 / \mu_{V E}\right)$ in the non facet-to-facet case. The following class of models reveal that $\mu_{E P}$ is indeed unbounded when $\mu_{V E}=4$.

- Example(s) 14: Column tessellations. Given a stationary planar tessellation $\mathcal{Y}^{\prime}$ we construct a spatial tessellation as follows. Each cell of $\mathcal{Y}^{\prime}$ is the base of an infinite cylinder perpendicular to the plane $\mathcal{E}$ in which $\mathcal{Y}^{\prime}$ lies. These cylinders are called columns. They pack to fill $\mathbb{R}^{3}$. Now any column is intersected by planes parallel to $\mathcal{E}$ with constant separation 1 . The position of these cuts is stationary and independent of those in the neighboring columns (unlike the positioning in the stratum models which aligned the cuts in all columns).

The result is a stationary spatial tessellation - the column tessellation $\mathcal{Y}$. Any cell of $\mathcal{Y}$ is a right prism with height 1 and a base facet which is a translate of a cell of $\mathcal{Y}^{\prime}$. Due to the independence of cuts in the columns, no column tessellations are facet-to-facet.

The topology of $\mathcal{Y}$ is determined by the topology of $\mathcal{Y}^{\prime}$. Hence the seven parameters for a column tessellation are functions of some topological parameters of the planar tessellation. Besides $\mu_{V E}^{\prime}$ and $\phi$ we need

$\circ \mu_{E V[\pi]}^{\prime}:=$ the mean number of $\pi$-vertices adjacent to the typical edge and

$\circ \mu_{V E}^{\prime(2)}:=$ the second moment of the number of edges adjacent to the typical vertex.

We obtain

$$
\begin{array}{rlrl}
\mu_{V E}=4 & \xi & =\frac{1}{2}+\frac{1}{4} \mu_{E V[\pi]}^{\prime} \\
\mu_{E P}=\frac{1}{2 \mu_{V E}^{\prime}}\left(3 \mu_{V E}^{\prime}+\mu_{V E}^{\prime(2)}\right) & \kappa & =\frac{1}{2} \mu_{E V[\pi]}^{\prime}-\frac{\phi}{\mu_{V E}^{\prime}} \\
\mu_{P V}=\frac{2}{3 \mu_{V E}^{\prime}-2}\left(3 \mu_{V E}^{\prime}+\mu_{V E}^{\prime(2)}\right) & \psi & =\frac{\mu_{V E}^{\prime(2)}+3 \phi}{\mu_{V E}^{\prime}}-1-\frac{1}{2} \mu_{E V[\pi]}^{\prime} \\
\tau & =\frac{\mu_{V E}^{\prime(2)}+\phi}{\mu_{V E}^{\prime}}-2 .
\end{array}
$$

The proof of these identities is available in [10]. Their use can be seen by looking again at Examples 6(a)-6(d). As an exercise, one can show that Example 6(c) obeys these identities; by using the two-dimensional entities $\mu_{V E}^{\prime}=\frac{10}{3}, \mu_{V E}^{\prime(2)}=\frac{34}{3}$ and $\phi=\mu_{E V[\pi]}^{\prime}=0$, one recovers the cited three-dimensional answers.

The following column tessellation is based on a planar tessellation whose second moment $\mu_{V E}^{\prime(2)}$ is unbounded and, from that observation, we establish that the spatial model has no upper bound for $\mu_{E P}$. The starting point is a stationary tessellation in $\mathbb{R}^{2}$, where all cells are unit squares which are positioned side-to-side. Now each cell is divided by a further 
vertex in its relative interior, the vertex having $4 n$ emanating edges, $n$ of them to each side of the square, $n \geq 1$. Moreover those interior edges of two squares having a common side are disjoint; they do not meet at a common vertex in the interior of that common side. Hence any square side has $2 n$ new vertices in its relative interior - and they are all $\pi$-vertices with three emanating edges.

For such a planar tessellation we obtain

$$
\begin{aligned}
\mu_{V E}^{\prime}= & \frac{1 \cdot 4+2 \cdot 2 n \cdot 3+1 \cdot 4 n}{1+2 \cdot 2 n+1}=\frac{2(4 n+1)}{2 n+1} & \phi & =\frac{2 n}{2 n+1} \\
\mu_{V E}^{\prime(2)} & =\frac{1 \cdot 16+2 \cdot 2 n \cdot 9+1 \cdot 16 n^{2}}{1+2 \cdot 2 n+1}=\frac{2\left(4 n^{2}+9 n+4\right)}{2 n+1} & \mu_{E V[\pi]}^{\prime} & =\frac{6 n}{4 n+1} .
\end{aligned}
$$

Hence $\mu_{E P}$ for column tessellations is unbounded and all the tessellations from that model lie on the line $\mu_{V E}=4$ with $\mu_{E P} \geq 4$ (see Figure 1 where the point which represents Example 14 has $n=8$ and $\left.\left(\mu_{V E}, \mu_{E P}\right)=\left(4, \frac{431}{66}\right)\right)$.

REMARK 5: Note that every point $Q$ on the curve $\mu_{E P}=6\left(1-2 / \mu_{V E}\right)$ represents some tessellation. This is obvious from our earlier theory of mixture curves if $Q$ lies on the curve between the three tessellations represented by $(4,3)$ and some point $R$ on the curve. For illustration, taking $R$ as the point from Example 2, we conclude that every point $Q$ on the curve between $(4,3)$ and $R$ represents a tessellation because the curve is of the generic form $\mu_{E P}=A-B / \mu_{V E}$ closed under the mixture operation. There is always such a $R$, however, for every such $Q$. This is so, because for any $R$ higher on the curve than $\left(\mu_{V E}, \mu_{E P}\right)$ of Example 2 , can be achieved by mixture. One can always find a column tessellation represented by $\left(4, \mu_{E P}^{[1]}\right)$ for some suitably large $\mu_{E P}^{[1]}$ and a tessellation based on Example 11 with a suitably large $\mu_{V E}^{[2]}$ such that their mixture curve passes through $R$.

REMARK 6: Since $\mu_{E P}$ is unbounded when $\mu_{V E}=4$, we can use the mixture concept and Remark 5 to establish that $\mu_{E P}$ is unbounded for all $\mu_{V E}$.

When $\xi>0$, other interior parameters can be zero: For facet-to-facet tessellations, we know that $\xi=\kappa=\psi=\tau=0$. We also know that $\xi>0$ if and only if the tessellation is not facet-to-facet. The other interior parameters, $\kappa, \psi$ and $\tau$, can still be zero when $\xi>0$, as the following two examples show.

- Example 15: Start with the cubic lattice of Example 5, aligned to the three Cartesian axes. Divide each cube into two congruent triangular prisms using a rectangular plate. Vary the orientation of the plate, as shown in Figure 4(c). Clearly every such plate creates two $\pi$-edges in the resulting tessellation. It is easy to show that $\left(\mu_{V E}, \mu_{E P}\right)=$ $(10,4), \mu_{P V}=\frac{10}{3}, \xi=\frac{2}{5}$ and $\kappa=\psi=\tau=0$.

- Example 16: Also start with the aligned cubic lattice. Partition each cube into three congruent pyramids as per Figure 4(a), with all of the $A B$ chords (one for each cube) being parallel and with all points marked $A$ being at the low end of the chord. Thus each cube-facet with a dividing diagonal is next to one with no such diagonal. These diagonals are therefore $\pi$-edges in the new tessellation. As they represent $\frac{3}{7}$ of all edges, $\xi=\frac{3}{7}$. No vertices lie in the interior of any facet, ridge or plate-side, so $\kappa=\psi=\tau=0$. For the record, $\left(\mu_{V E}, \mu_{E P}\right)=\left(14, \frac{27}{7}\right)$ and $\mu_{P V}=3$.

When $\xi>0$ it is possible that $\psi>0$ and $\kappa=0$ (Examples 6, 8 and 9) or that $\kappa>0$ and $\psi=0$ (Example 17 below). 
- Example 17: Adjust each column in Example 6a so that the triangular facets are aligned on parallel planes. There is now no offset between columns and we have a stratum tessellation. In every second stratum, partition every cell into three congruent prisms as shown in Figure 7(a). The new vertices created are all hemi-vertices and one can show that $\kappa=\frac{2}{3}$ and $\psi=\tau=0$. Also $\xi=\frac{6}{11},\left(\mu_{V E}, \mu_{E P}\right)=\left(\frac{22}{3}, \frac{42}{11}\right)$ and $\mu_{P V}=\frac{7}{2}$.

\section{Books with spines}

Three of the interior parameters, $\kappa, \psi$ and $\tau$, are related to vertices being in the interior of ridges, plate-sides or facets, whereas the other interior parameter $\xi$ is related to $\pi$-edges. To detect constraints for the interior parameters it makes sense to utilize relations between $\pi$-edges and interior vertices based on the combinatorial topology of the tessellation. There is a wide variety of arrangements and, to handle all those which are of interest, we use a new concept: books with their spines. We introduce the concept by explaining how plate-sides whose interior contains some vertex $v$ can form the pages of a book.

Definition 1: Let $v$ be a vertex and $\ell$ be a line passing through $v$. So $v \subset \ell$. We define a page as a plate which has one of its sides $\subset \ell$ and the interior of that side $\supset v$. A book-cover is a cell-facet which has one of its sides $\supset v$ and contains $k \geq 2$ plates that have a corner coinciding with $v$. Note: Exactly two of these plates have a side that is $\subset \ell$. Furthermore, the facet contains $(k-1) \pi$-edges that emanate from $v$ and it may have other plates and $\pi$-edges that are not adjacent to $v$.

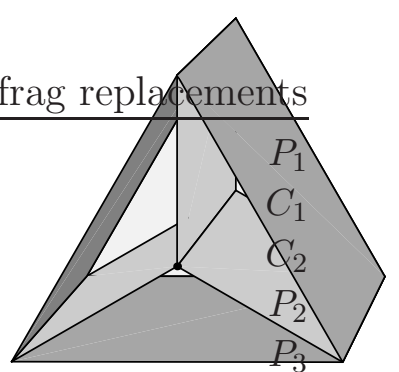

(a)

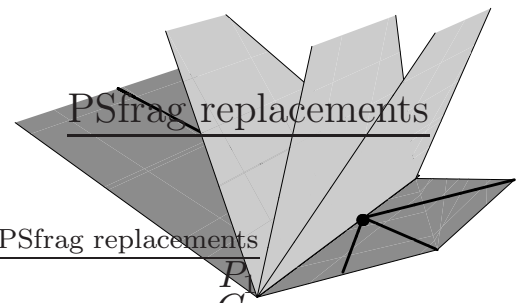

(b)

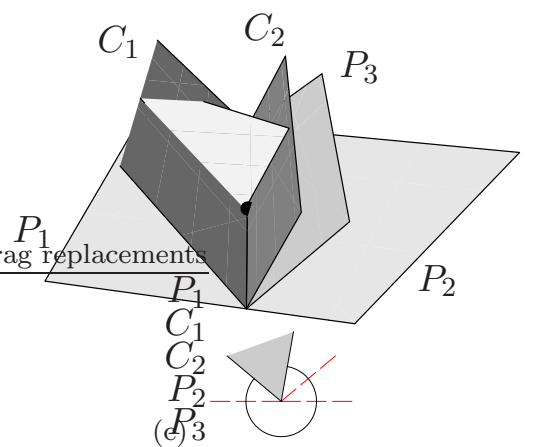

(c)

Figure 7: (a) All cells in every second stratum of Example 17 are partitioned as shown, into three congruent triangular prisms. (b) A 3-page book with its two book-covers in darker shading. There are always edges on the book-covers emanating from the vertex which is on the book's 'spine' (c) A vertex adjacent to three plate-side-interiors, three ridge-interiors and one facet-interior (the horizontal plates $P_{1}$ and $P_{2}$ forming in union a facet of a cell below). Note: if the light-coloured plate between the two dark structures $C_{1}$ and $C_{2}$ and with a corner touching the vertex were removed, then the vertex would not exist. Using the 'book' terminology, we see pages $P_{1}, P_{2}$ and $P_{3}$ and two bookcovers $C_{1}$ and $C_{2}$.

In Figure 7(b), three pages and two darkly-shaded book-covers (the fully visible one having $k=4)$ are shown, in a neighbourhood of a vertex. The overall appearance is like a book.

Definition 2: At vertex $v$, a book with $p \geq 0$ pages is a collection $\mathcal{B}$ comprising p pages and two book-covers in such a way that a circular arc, with small radius and centred at $v$, can be drawn from one bookcover to the other encountering no plates other than those which form the p pages. The line $\ell$ mentioned above is called the spine of the book.

REMARK 7: It is time to describe the schematic figures, which look at a vertex $v$ along the spine. The bookcovers are black whilst the pages are dashed and red. The circular arc is shown and shaded sectors represent the plate (or plates) that have a corner on $v$, thus ensuring that $v$ 

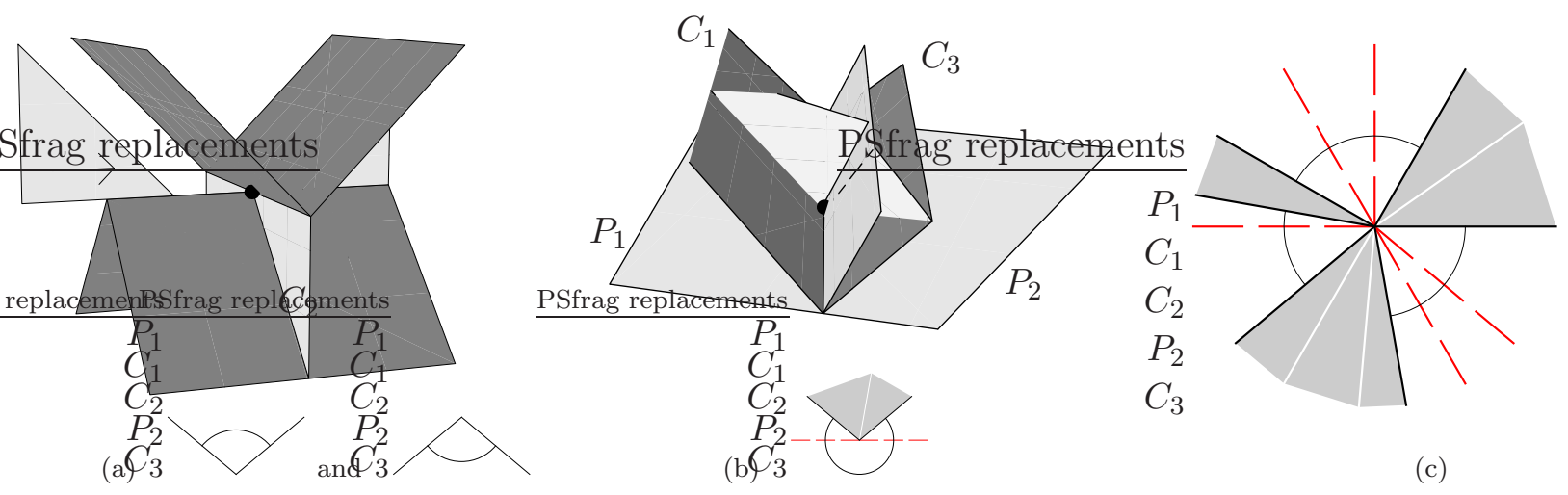

(c)

Figure 8: (a) Two-books associated with a common vertex and with different spines. (b) A hemi-vertex $v$ in the interior of a facet made from the coplanar pages $P_{1}$ and $P_{2}$. There is one book, with two pages. The additional architecture has two plates with corners touching $v$ and both of these plates have created a $\pi$-edge on the grey structure that follows the spine. (c) The schematic diagram for a vertex having three books each with a common spine.

is indeed a vertex. Figure 7(c) provides a simple example. In Figure 8(c), which schematically shows three books with a common spine, we see shaded sectors sub-divided by white lines. Such a white line, also evident in Figure 8(b), represents a coplanar structure that 'lies along' the spine in a neighbourhood of $v$, but a structure that is neither a page nor a bookcover.

If $v$ is a hemi-vertex, then there may be a book with two co-planar pages that are encountered consecutively as one moves along the arc; Figure 7(c) has a 3-page book like this, comprising pages $P_{1}, P_{2}$ and $P_{3}$ and bookcovers $C_{1}$ and $C_{2}$. There may also be a hemi-vertex's book that has co-planarity of a page and a bookcover encountered on the arc immediately before or after the page; see Figure 2(c) where page $P$ and bookcover $C_{1}$ are coplanar. A non-hemi vertex cannot have a book with such consecutive coplanarity, although non-consecutive coplanarity is possible. (Figure 2(b) has two books each with coplanar, non-consecutive, bookcovers.)

Lemma 2: If a vertex $v$ has a p-page book associated with it, then $v$ lies in the interior of $p+1$ ridges of cells and in the interior of $p$ sides of plates that lie within the book, with the exception that a hemi-vertex having one of the 'consecutive coplanarity' structures mentioned above is adjacent to $p$ such ridge-interiors and $p$ such plate-side-interiors.

PROOF: (i) Each page of the book is a plate of the tessellation with $v \subset$ an interior of a side of the plate and this side $\subset \ell$. A plate on a bookcover either has a corner that coincides with $v$ or does not contain $v$. Therefore $v$ lies in the interior of $p$ plate-sides within the book. (ii) As one moves along the arc from one bookcover to the other, one encounters $p+1$ cells of the tessellation. If there is no 'consecutive coplanarity', then each of these cells has a ridge $\subset \ell$, with $v$ being in the interior of that ridge. If there is a consecutive coplanarity, and there can be at most one, the cell involved does not have a ridge $\subset \ell$ and therefore $v$ is in the interior of just $p$ ridges.

REMARK 8: A book without pages is possible, but then the dihedral angle between the two bookcovers must be $<\pi$, thereby guaranteeing a ridge along the spine. In such a structure, the bookcovers are consecutively encountered on the arc, of course. If we increased the dihedral angle to $\pi$, then this ridge disappears, the book ceases to exist and the erstwhile bookcovers (though still considered to be consecutive) cease individually to be facets and therefore lose the status of bookcovers. In summary, it is not possible to have a book with no pages and two coplanar bookcovers. 
Note that a vertex may have no books associated with it. For example, none of the vertices in a facet-to-facet tessellation have associated books and many of our non facet-to-facet examples have non-book vertices; every vertex in the stratum models of Example 9 has no associated book. Of course, a vertex with no books is not contained in any ridge-interior (and vice versa).

Vertices can have more than one book. The total numbers, $m_{Z_{1}}^{\circ}(v)$ and $m_{P_{1}}^{\circ}(v)$, of ridgeinteriors and plate-side-interiors adjacent to $v$ is simply the addition of such adjacencies over all books associated with $v$. An example is the schematic of Figure 8 (c), where $m_{Z_{1}}^{\circ}(v)=3+2+3$ and $m_{P_{1}}^{\circ}(v)=2+1+2$.

Multi-book vertices have many variants. For example, Figure 2(b) shows a $(p=2)$-book above the plane of the horizontal plates and an obliquely-oriented $(p=1)$-book below with a subtle sharing of the horizontal plates in the making of book-covers. This sharing can only occur when the books have different spines. A hemi-vertex can have multiple-books, though all with a common spine. For example in Figure $7(\mathrm{c})$, insert a plate in between pages $P_{2}$ and $P_{3}$

with a corner touching $v$, converting spine, one of the books with no pages and the other with $P_{1}$ as its sole page.

In general, the books (if any) for a non-hemi vertex can be mutually oblique; the generic situation for a non-hemi vertex $v$ with $n$ books is as follows. Draw $n$ lines of differing orientations passing through $v$. On each line construct a book initially with no pages using the line as spine. The dihedral angle between the covers will be small if $n$ is large. To increase $p$ for some of these books, just add further pages.

Figure 8(a) shows an example where $n=2$; the books, each with $p=0$, are shown with dark shading and one possible version of the surrounding architecture is being assembled (the fourth triangular plate almost in position).

Although the additional architecture around $v$ and its books can be very complicated, beyond our powers of visualisation and analysis if $n$ is large and many spines are involved, we know that no contributions to $m_{Z_{1}}^{\circ}(v)$ and $m_{P_{1}}^{\circ}(v)$ come from this architecture. The simple additive count over books suffices.

Even when $n=1$, the additional architecture can be complicated. In Figure 7 (c) it is simple, but if that figure is modified by inserting a plate in between page $P_{3}$ and book-cover $C_{2}$ with a corner touching $v$, it is more elaborate (as shown in Figure 8(b)). The modification has turned $P_{3}$ into a book cover (now labelled $C_{3}$ ) which, together with the other book-cover $C_{1}$ and the pages $P_{1}$ and $P_{2}$, form a book (the only book associated with $v$ ). The additional architecture comprises the two plates each with a corner touching $v$ and the structure we previously labelled $C_{2}$, although this is no longer a book-cover.

\section{Constraints on the interior parameters $\xi, \kappa, \psi$ and $\tau$}

The considerations in the last section can be applied to achieve inequalities involving the interior parameters; we do so in Lemma 3 below. Part of this lemma, formula (17), establishes one of the bounds for $(\kappa, \xi)$ given in Theorem 1. Furthermore, formula (16) provides a component to both the upper bound and lower bound of inequality (8) in that theorem. 
LEMMA 3: For stationary random tessellations of $\mathbb{R}^{3}$ that are not facet-to-facet,

$$
\begin{aligned}
& 0 \leq \psi-\tau \leq \frac{1}{2} \mu_{V E} \quad \text { and } \\
& \xi \geq \frac{2(\psi-\tau)+3 \kappa}{\mu_{V E}}=L 1(\text { say }),
\end{aligned}
$$

augmenting the basic condition $(\kappa, \xi) \in[0,1] \times(0,1]$ which follows from the definitions. Note: the label L1 identifies this as the first of two lower bounds that we establish for $\xi$.

PRoOF: We can readily conclude that $\tau \leq \psi$ using Lemma 2 which shows that, for every book associated with the vertex $v$, the number of adjacent plate-side-interiors is $\leq$ the number of adjacent ridge-interiors. From the discussion in Section 7 , we have $m_{P_{1}}^{\circ}(v) \leq m_{Z_{1}}^{\circ}(v)$ for all $v$. Taking expectations for typical $v$ shows that $\tau \leq \psi$.

To prove (17), we use $\xi \mu_{V E}=\mu_{V E[\pi]}$, see (41), and show that, for a vertex $v \in V$, there are lower bounds on $m_{E[\pi]}(v)$, the number of $\pi$-edges adjacent to (that is, emanating from) the vertex $v$.

$$
m_{E[\pi]}(v) \geq \begin{cases}2\left(m_{Z_{1}}^{\circ}(v)-m_{P_{1}}^{\circ}(v)\right) & \text { if } v \text { is not a hemi }- \text { vertex }, \\ 4+2\left(m_{Z_{1}}^{\circ}(v)-m_{P_{1}}^{\circ}(v)\right) & \text { if } v \text { is a hemi }- \text { vertex and } m_{Z_{1}}^{\circ}(v)>0, \\ 3 & \text { if } v \text { is a hemi }- \text { vertex and } m_{Z_{1}}^{\circ}(v)=0 .\end{cases}
$$

To show these inequalities, first let $v$ be a non-hemi vertex. With Lemma 2 the number of books that $v$ has is $m_{Z_{1}}^{\circ}(v)-m_{P_{1}}^{\circ}(v)$, because none of these books have consecutive coplanarity. Every book-cover contributes at least one $\pi$-edge to the count of $m_{E[\pi]}(v)$. Outside these books there are no further ridge or plate-side interiors containing $v$. The arrangement changing Figure 7(c) to Figure 8(b) (that is, - to - affects no change of $m_{Z_{1}}^{\circ}(v)-m_{P_{1}}^{\circ}(v)$, whereas the number of $\pi$-edges adjacent to $v$ can increase; it increases by two in Figure 8 (b), but this could be more with complicated structures and it might not increase at all (as can be imagined if the two white plates in Figure 8(b) were aligned). This proves the first inequality in (18).

Now consider $v$ as a hemi-vertex with one spine and $n \geq 1$ books. If none of these books are consecutive coplanar, eliminating for example

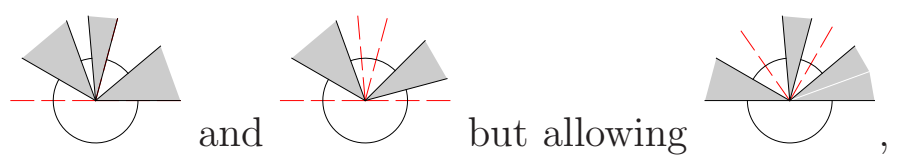

the number of $\pi$-edges emanating from $v$ and contained in the bookcovers is again $\geq 2\left(m_{Z_{1}}^{\circ}(v)-\right.$ $\left.m_{P_{1}}^{\circ}(v)\right)$. The facet containing the hemi-vertex $v$ contains at least four $\pi$-edges adjacent to $v$ (two on the spine and at least two off the spine). If one of the books is consecutive coplanar the number of books now is $m_{Z_{1}}^{\circ}(v)-m_{P_{1}}^{\circ}(v)+1$. The facet containing $v$ has at least two $\pi$-edges emanating from $v$, see Figure $7(\mathrm{c})$.

A hemi-vertex with no books must have three or more emanating $\pi$-edges lying in the interior of the facet that contains $v$. Now (18) is fully proven.

Inequalities (18) can be written as:

$$
m_{E[\pi]}(v) \geq \begin{cases}3 m_{Z_{2}}^{\circ}(v)+2\left(m_{Z_{1}}^{\circ}(v)-m_{P_{1}}^{\circ}(v)\right) & \text { if } v \text { is not a hemi }- \text { vertex }, \\ 1+3 m_{Z_{2}}^{\circ}(v)+2\left(m_{Z_{1}}^{\circ}(v)-m_{P_{1}}^{\circ}(v)\right) & \text { if } v \text { is a hemi }- \text { vertex and } m_{Z_{1}}^{\circ}(v)>0, \\ 3 m_{Z_{2}}^{\circ}(v)+2\left(m_{Z_{1}}^{\circ}(v)-m_{P_{1}}^{\circ}(v)\right) & \text { if } v \text { is a hemi }- \text { vertex and } m_{Z_{1}}^{\circ}(v)=0 .\end{cases}
$$


So, for general $v$,

$$
m_{E[\pi]}(v) \geq 2\left(m_{Z_{1}}^{\circ}(v)-m_{P_{1}}^{\circ}(v)\right)+3 m_{Z_{2}}^{\circ}(v) .
$$

Therefore,

$$
\mu_{V E[\pi]} \geq 2(\psi-\tau)+3 \kappa .
$$

With (44) we prove (17). Inequality (17) leads to the proof of the upper bound in (16).

Known results involving $\xi, \kappa, \psi$ and $\tau$ : Some of the most important results in our earlier paper, [15], are three formulae that yield the expected numbers of apices, ridges and facets for a typical cell - entities that are equal to the mean adjacencies $\mu_{Z V}, \mu_{Z E}$ and $\mu_{Z P}$ (given in Table 1) in the facet-to-facet case, but not in general. These three formulae follow:

$$
\begin{aligned}
& \nu_{0}(Z)=\mu_{Z V}-\mu_{P V} \frac{2(\kappa+\psi)}{f\left(\mu_{P V}\right)} ; \\
& \nu_{1}(Z)=\mu_{Z E}-\mu_{P V} \frac{\xi \mu_{V E}+2 \psi}{f\left(\mu_{P V}\right)} ; \\
& \nu_{2}(Z)=\mu_{Z P}-\mu_{P V} \frac{\xi \mu_{V E}-2 \kappa}{f\left(\mu_{P V}\right)} .
\end{aligned}
$$

Our earlier paper also presented formulae for the mean number of sides (also of corners) for the typical cell-facet and for the typical plate.

$$
\begin{aligned}
\nu_{1}\left(Z_{2}\right) & =\nu_{0}\left(Z_{2}\right)=2 \mu_{P V} \frac{\mu_{V E}\left(\mu_{E P}-\xi\right)-2 \psi}{\left.2 \mu_{V E} \mu_{E P}-\mu_{P V}\left(\xi \mu_{V E}-2 \kappa\right)\right)} \\
\nu_{1}(P) & =\nu_{0}(P)=\mu_{P V}\left(1-\frac{2 \tau}{\mu_{V E} \mu_{E P}}\right) .
\end{aligned}
$$

Intensities, shown in Table 2 below, were also given there. These augment the list of intensities for the primitive elements given in Table 1.

\begin{tabular}{|c||c|}
\hline$X$ & $\lambda_{X} / \lambda_{V}$ \\
\hline \hline facets $Z_{2}$ & $\frac{2 \mu_{V E} \mu_{E P}-\mu_{P V}\left(\xi \mu_{V E}-2 \kappa\right)}{2 \mu_{P V}}$ \\
ridges $Z_{1}$ & $\frac{1}{2}\left(\mu_{V E}\left(\mu_{E P}-\xi\right)-2 \psi\right)$ \\
apices $Z_{0}$ & $\frac{1}{2} f(2)-\kappa-\psi$ \\
\hline facet-sides $\left(Z_{2}\right)_{1}$ or facet-corners $\left(Z_{2}\right)_{0}$ & $\mu_{V E}\left(\mu_{E P}-\xi\right)-2 \psi$ \\
\hline plate-sides $P_{1}$ or plate-corners $P_{0}$ & $\frac{1}{2}\left(\mu_{V E} \mu_{E P}-2 \tau\right)$ \\
\hline
\end{tabular}

Table 2: Intensities for non-primitives in spatial tessellations. Note the iterated notation, for example $\left(Z_{2}\right)_{1}$ for facet-sides.

We have systematically examined every sensible inequality that can be applied to these formulae. Firstly, we investigated the constraints needed on our seven parameters to ensure that all intensities from Tables 1 and 2 are non-negative (and in some cases, positive). Secondly we have examined the consequence of the inequalities $\nu_{0}(Z) \geq 4, \nu_{1}(Z) \geq 6, \nu_{2}(Z) \geq 4, \nu_{1}\left(Z_{2}\right) \geq 3$ and $\nu_{1}(P) \geq 3$. We do not report details of the constraints from the first exercise as these were 
dominated by those from the second, which yield as follows.

$$
\begin{aligned}
& \nu_{0}(Z) \geq 4 \Longleftrightarrow \mu_{Z V}-\mu_{P V} \frac{2(\kappa+\psi)}{f\left(\mu_{P V}\right)} \geq 4 \\
& \Longleftrightarrow \kappa \leq \mu_{V E}-2+\frac{1}{2} \mu_{V E} \mu_{E P}\left(1-\frac{4}{\mu_{P V}}\right)-\psi=K \text { (say). } \\
& \text { Likewise, } \nu_{1}(Z) \geq 6 \quad \Longleftrightarrow \xi \leq 6\left(1-\frac{2}{\mu_{V E}}\right)+\mu_{E P}\left(1-\frac{6}{\mu_{P V}}\right)-\frac{2 \psi}{\mu_{V E}}=U 1 \text { (say), } \\
& \nu_{2}(Z) \geq 4 \quad \Longleftrightarrow \xi \leq 4\left(1-\frac{2}{\mu_{V E}}\right)-\frac{2 \mu_{E P}}{\mu_{P V}}+\frac{2 \kappa}{\mu_{V E}}=U 2(\text { say }) \\
& \text { and } \nu_{1}\left(Z_{2}\right) \geq 3 \quad \Longleftrightarrow \xi \geq \frac{4 \psi+6 \kappa}{\mu_{V E}}-2 \mu_{E P}\left(1-\frac{3}{\mu_{P V}}\right)=L 2 \text { (say). }
\end{aligned}
$$

In the non facet-to-facet theory, the basic identity (9) applicable to each of the tessellation's convex cells becomes $2 \nu_{1}(Z) \geq 3 \nu_{0}(Z)$ and this is equivalent to

$$
\xi \leq 3-\frac{\mu_{E P}}{2}+\frac{\psi-6+3 \kappa}{\mu_{V E}}=U 3 \text { (say), }
$$

an identity which can also be derived from $3 \lambda_{Z} \nu_{0}(Z) \leq \lambda_{Z_{2}} \nu_{0}\left(Z_{2}\right)$. The other polyhedral inequality (10) yielded $2 \nu_{1}(Z) \geq 3 \nu_{2}(Z)$ but this led to something already known, namely (27).

Constraints on $\xi$ and $\kappa$, given $\mu_{V E}, \mu_{E P}, \mu_{P V}, \psi$ and $\tau$ : We see in (17) and (24)-(28) two lower bounds and three upper bounds for $\xi$, plus one upper bound for $\kappa$. The bounds are expressed in terms of $\mu_{V E}, \mu_{E P}, \mu_{P V}, \psi$ and $\tau$ which, for the moment pending further investigation, are assumed to be 'appropriate' — permitting a non-null set of $(\kappa, \xi)$ values.

Considerable insight on the permitted $(\kappa, \xi)$ values comes from Figure 9, where the bounds appear as straight lines — because the bounds on $\xi$ are linear in $\kappa$.

LEMMA 4: The permitted range for $\kappa$ and $\xi$, given $\mu_{V E}, \mu_{E P}, \mu_{P V}, \psi$ and $\tau$, is given by $0 \leq \kappa \leq \min [1, K], \xi>0$ and $\max (L 1, L 2) \leq \xi \leq \min [1, U 3]$, written in full form as

$$
\begin{aligned}
& 0 \leq \kappa \leq \min \left[1, \mu_{V E}-2+\frac{1}{2} \mu_{V E} \mu_{E P}\left(1-\frac{4}{\mu_{P V}}\right)-\psi\right] \quad \text { and } \\
& \max \left[\frac{2(\psi-\tau)+3 \kappa}{\mu_{V E}}, \frac{4 \psi+6 \kappa}{\mu_{V E}}-2 \mu_{E P}\left(1-\frac{3}{\mu_{P V}}\right)\right] \leq \xi \leq \min \left[1,3-\frac{\mu_{E P}}{2}+\frac{\psi-6+3 \kappa}{\mu_{V E}}\right],
\end{aligned}
$$

supplemented by $\xi>0$.

Proof: Figure 9 suggests that five of the straight lines pass through a common point, labelled $C$, whose coordinates are obviously $(\kappa, \xi)=(K, U 1)$. It can be proved generally with simple algebra that $C$ lies on the other three lines $U 2, U 3$ and $L 2$ (details omitted). The redundancy of $U 1$ and $U 2$ follows, because the line for $U 3$ has a higher slope than those for $U_{1}$ and $U_{2}$, implying $U_{1} \geq U_{3}$ and $U_{2} \geq U_{3}$ when $\kappa \leq K$ (as it always is, from (24) ).

Bound $L 1$ is established in Lemma 3. The redundancy of $U 1$ and $U 2$, leaves only (24),(27) and (28), and they provide the remaining bounds to complete the lemma.

Note that the permitted zone for $(\kappa, \xi)$ can have zero area, as in Figure 9(b) where the zone is a line-segment or in Example 8, the Divided Delaunay tessellation, where one can show that the zone is the single point $(\kappa, \xi)=\left(0,64 \pi^{2} /\left(35+112 \pi^{2}\right)\right) \approx(0,0.554)$.

Figure 9(f) raises an interesting issue. The lines $L 1$ and $U 3$ can never cross because they are parallel. Yet, the values chosen for $\mu_{V E}, \mu_{E P}, \mu_{P V}, \psi$ and $\tau$ have created the situation where 

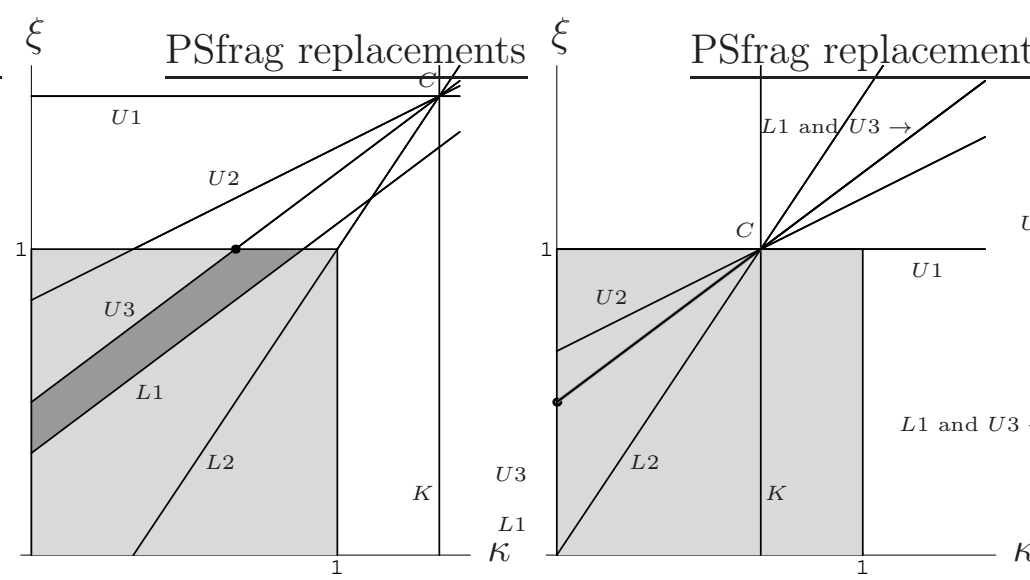

(a) Example 4, STIT

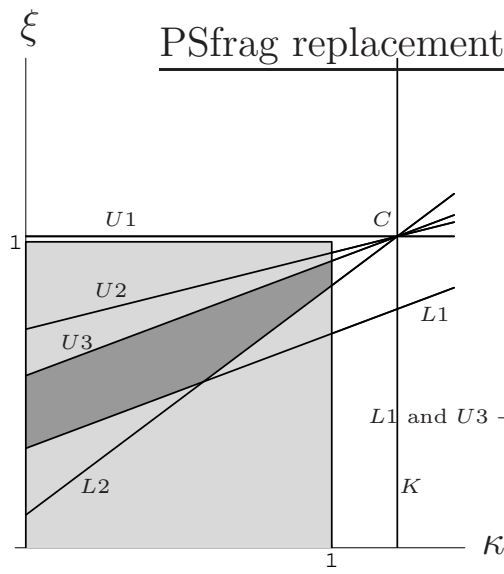

(b) Example 6a, triangular prism model

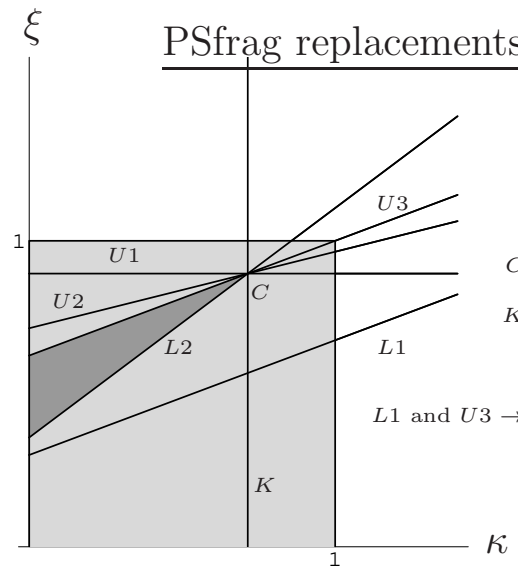

(e) $L 1$ has no impact. Also $C \in[0,1] \times(0,1]$

(d) $L 2$ impacts on zone

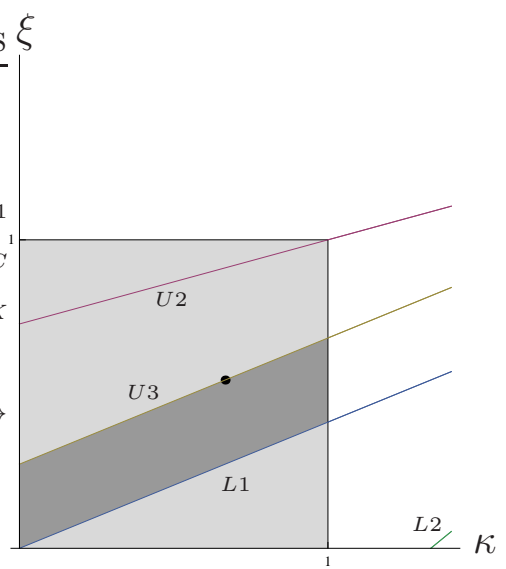

(c) Example 17

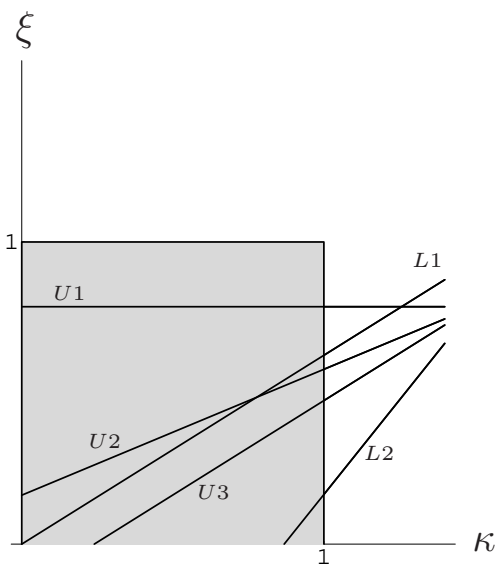

(f) Null zone

Figure 9: Examples of permitted zones for $(\kappa, \xi)$ shown in the darker shading, against the light grey region $[0,1] \times(0,1]$. In (a)-(c), three of our examples are featured, whilst (d)-(f) show other variations. For (a)-(c), the heavy dot shows the $(\kappa, \xi)$ point of that example whilst the dark shading shows the permitted range of $(\kappa, \xi)$ for tessellations which have the same $\mu_{V E}, \mu_{E P}, \mu_{P V}$ and $\psi$ values as the model which produced the heavy dot. In (b), the darker zone is a line-segment running between the heavy dot and $C$. In (f) the zone is null because the lower bound $L 1$ is higher than the upper bound $U 3$, implying that the choice of $\mu_{V E}, \mu_{E P}, \mu_{P V}, \psi$ and $\tau$ is inappropriate. For the record, (d) and (e) use $\mu_{V E}=8, \mu_{E P}=4$ and $\mu_{P V}=\frac{7}{2}\left(\right.$ with $(\psi, \tau)=\left(\frac{5}{2}, \frac{6}{5}\right)$ for $(\mathrm{d})$ and $(\psi, \tau)=\left(3, \frac{9}{5}\right)$ for $\left.(\mathrm{e})\right)$. In (f), $\mu_{V E}=\frac{24}{5}, \mu_{E P}=\frac{19}{5}$ and $\mu_{P V}=\frac{7}{2}$, with $\psi=\tau=0$.

$L 1>U 3$. This is clearly not allowed, so something must be inappropriate in the choice of $\mu_{V E}, \mu_{E P}, \mu_{P V}, \psi$ and $\tau$. This issue motivates our next subsection.

Appropriate values for $(\psi, \tau)$ : We must avoid a choice of $\psi$ and $\tau$ which leads to $L 1>U 3$. For a tessellation to exist, we require $L 1 \leq U 3$ which after rearrangement becomes

$$
\tau \geq \frac{\psi}{2}+\frac{\mu_{V E}}{4}\left(\mu_{E P}-6\left(1-\frac{2}{\mu_{V E}}\right)\right)
$$

This is the first example of a constraint on $(\psi, \tau)$ arising from the need to avoid a null space for $(\kappa, \xi)$. Other such constraints follow shortly, but firstly we consider one other inequality which applies for $\tau$. From inequality (23), which represents the requirement $\nu_{1}(P) \geq 3$, we have that

$$
\tau \leq \frac{1}{2} \mu_{V E} \mu_{E P}\left(1-\frac{3}{\mu_{P V}}\right)
$$

The bound (30) is the last inequality (used in conjunction with (16) of Lemma 3) needed to complete our proof of (8) in Theorem 1. 
REMARK 9: Consider again (29). Note that the term in the larger brackets relates to the fundamental curve of Figure 1. So, the tessellation is represented by a point above the curve in Figure 1, if and only if $\tau>0$. Thus the only tessellations below the curve have $\tau=0$. These findings were anticipated in the caption of Figure 5.

There does not exist a tessellation if $K<0$ or if $U 1<0$ and this fact constrains $\psi$ somewhat. From (24) and (25), we have respectively two constraints:

$$
\begin{aligned}
& \psi \leq \mu_{V E}-2+\frac{\mu_{V E} \mu_{E P}}{2}\left(1-\frac{4}{\mu_{P V}}\right) \\
& \psi \leq 3 \mu_{V E}-6+\frac{\mu_{V E} \mu_{E P}}{2}\left(1-\frac{6}{\mu_{P V}}\right) .
\end{aligned}
$$

Their violation would make the $(\kappa, \xi)$ domain null, so they are needed (or at least (31) is).

Lemma 5: For non facet-to-facet tessellations, the bound in (32) is greater than that in (31) and is therefore redundant.

PROOF: From (15), we know that $\mu_{P V}>\frac{1}{2} \frac{\mu_{V E} \mu_{E P}}{\mu_{V E}-2}$ when the tessellation is not facet-to-facet. Simple algebra shows that $\mu_{P V}>\frac{1}{2} \frac{\mu_{V E} \mu_{E P}}{\mu_{V E}-2} \Longleftrightarrow$ "bound of (32) $>$ bound of (31)".

We can also derive another upper bound for $\psi$ from (27). Although $L 2>U 3$ is not possible for any $\kappa$, so no tessellation-existence question arises in this way, there would be an issue if $\{\xi \geq L 2\} \cap\{(\kappa, \xi) \in[0,1] \times(0,1]\}=\emptyset$. In view of $L 2$ 's positive slope, it is enough to require that $L 2 \leq 1$ when $\kappa=0$ to avoid this problem and this yields the inequality

$$
\psi \leq \frac{\mu_{V E}}{4}+\frac{\mu_{V E} \mu_{E P}}{2}\left(1-\frac{3}{\mu_{P V}}\right)
$$

No further nullity-avoidance constraints are required, because Remark 10 below eliminates such issues with $L 1$ and $U 3$.

REMARK 10: With $0 \leq L 1 \leq 1$ when $\kappa=0$, the intersection of $\{(\kappa, \xi) \in[0,1] \times(0,1]\}$ and $\{\xi \geq L 1\}$ is non-empty. See six cases of L1 in Figure 9 which illustrate the line L1. If $\psi-\tau=\frac{1}{2} \mu_{V E}$, then the only permitted case is $(\kappa, \xi)=(0,1)$.

Simple algebraic manipulation establishes the following lemma which in turn establishes (7), thus completing the proof for another part of Theorem 1.

Lemma 6: Denote the right-hand sides of (31) and(33) by $R 1$ and $R 2$. Then

$$
\begin{aligned}
& R 2<R 1 \quad \Longleftrightarrow \quad \frac{2 \mu_{V E} \mu_{E P}}{3 \mu_{V E}-8}<\mu_{P V} \quad \text { and } \\
& R 1 \leq R 2 \quad \Longleftrightarrow \quad \mu_{P V} \leq \frac{2 \mu_{V E} \mu_{E P}}{3 \mu_{V E}-8}
\end{aligned}
$$

Moreover, $\frac{2 \mu_{V E} \mu_{E P}}{3 \mu_{V E}-8}$ lies within the permitted range for $\mu_{P V}$ shown in (6).

We conclude this subsection having established five constraints on the $(\psi, \tau)$ domain that have not been made redundant, namely $R 1$ and $R 2$ of Lemma 6 and (16), (29) and (30).

The permitted $(\psi, \tau)$ domain; an example plot: In parts $(\mathrm{d})$ and $(\mathrm{e})$ of Figure 9 are two examples sharing common values for the cyclic parameters, namely $\mu_{V E}=8, \mu_{E P}=4$ and $\mu_{P V}=\frac{7}{2}$. Figure 10(a) shows the allowed domain for $\left(\mu_{P V}, \mu_{E P}\right)$, given $\mu_{V E}=8$ (calculated from Theorem 1), together with the dot corresponding to the particular case. This drawing is in essentially the same format as the plots in Figure 5. The dashed line is an innovation, 
separating the domain into two parts, in keeping with Lemma 6 and (17). The particular dot lies to the left of this dashed line, indicating that $R 1<R 2$ and this is confirmed in Figure 10.(b), where $R 1$ and $R 2$ are marked.

In Figure 10(b), we show the allowed $(\psi, \tau)$ domain given the particular values of all three cyclic parameters. We see the all the constraints on $\tau$ and $\psi$ developed earlier in this section. The two dots in this domain are the values used in the preparation of Figure 9, with $(\psi, \tau)=$ $\left(\frac{5}{2}, \frac{6}{5}\right)$ for $(\mathrm{d})$ and $(\psi, \tau)=\left(3, \frac{9}{5}\right)$ for $(\mathrm{e})$. Note that each dot produces a qualitatively different $(\kappa, \xi)$ domain as seen in Figure 9 .

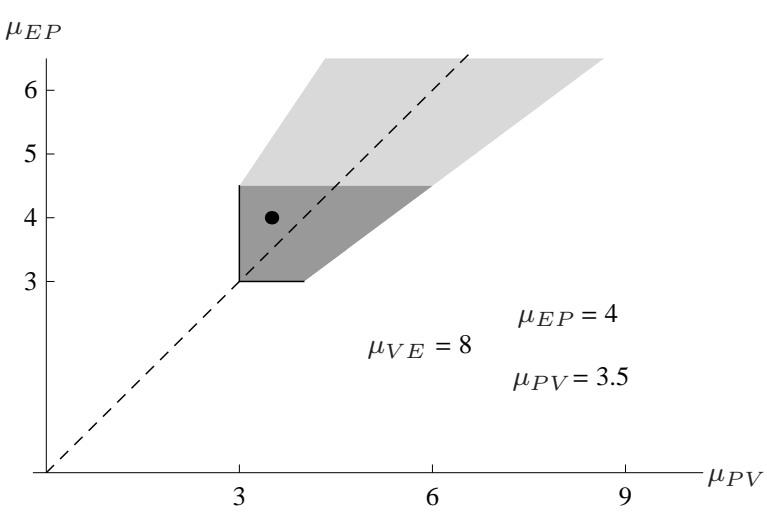

(a)

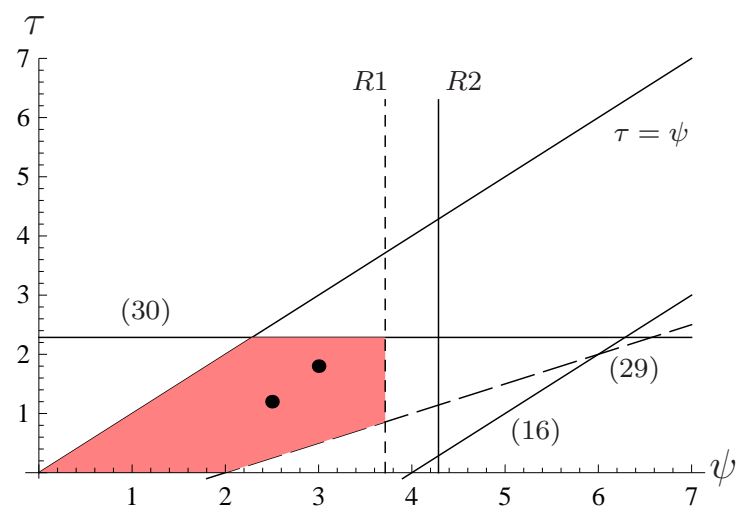

(b)

Figure 10: (a) This follows the style in Figure 5 except now we add the dashed line, whose equation is $\mu_{E P}=\frac{1}{2}\left(3 \mu_{V E}-\right.$ 8) $\mu_{P V} / \mu_{V E}$. The dot corresponds to $\left.\left(\mu_{P V}, \mu_{E P}\right)=\left(\frac{7}{2}, 4\right)\right)$. (b) The permitted domain for $(\psi, \tau)$ given $\left.\left(\mu_{P V}, \mu_{E P}\right)=\left(\frac{7}{2}, 4\right)\right)$, with the various bounds marked. See the text for further explanation and the connection with Figure 9

Are there further constraints on the cyclic parameters? We have not yet addressed the potential for the $(\psi, \tau)$ domain to be empty. Perhaps some values of $\mu_{V E}, \mu_{E P}$ and $\mu_{P V}$ which we have to date regarded as valid, create this null situation and lead to the non-existence of a tessellation? These concerns can, however, be dismissed.

Lemma 7: Cyclic parameters satisfying (6) in Theorem 1 cannot lead to a null domain for $(\psi, \tau)$.

PROOF: Simple algebraic calculations show that $\frac{1}{2} \mu_{V E} \mu_{E P}\left(1-3 / \mu_{P V}\right)$, which is the righthand side of (30), is less than $\min (R 1, R 2)$. It is also easy to show that $\frac{1}{2} \mu_{V E} \mu_{E P}\left(1-3 / \mu_{P V}\right)$ is greater than the maximum of the two lower bounds for $\tau$ given in (16) and (29) when both these bounds are evaluated at $\psi=\min (R 1, R 2)$. It is also obvious that $\min (R 1, R 2)>0$. The statements above hold for all values of the cyclic parameters which satisfy (6)). This proves that the domain for $(\psi, \tau)$ is not null under the stated premise of the lemma.

We conclude this section with the twelve diagrams that comprise Figure 11; in all diagrams $\mu_{V E}=7$. Also $\mu_{E P}$ increases as one goes down the page. Two values of $\mu_{P V}$ are shown on each row. A diversity of shapes for the $(\psi, \tau)$ permitted domain is demonstrated.

\section{Concluding remarks}

Although it is not easy to work in a seven-dimensional space, we think that our approach has produced an intelligible picture of the constraints that apply for spatial tessellations, in both the facet-to-facet case and the general case. 
We emphasise however that our focus has been combinatorial topology and our constraints are about topological parameters. We anticipate that further constraints will arise when geometric aspects are studied in greater detail.

Our approach to the topic of this paper has been rooted in the literature of random stationary tessellations of $\mathbb{R}^{3}$ and, although we allude to the tiling literature and present many examples which would be called 'tilings', we have not asked the same questions that tilers ask. We notice, however, that non facet-to-facet tilings of $\mathbb{R}^{3}$ have not often been studied in the literature, so we think that our findings have value for tilers.

We hope in later work to develop an interface between the two literatures. Tentative steps in that direction have shown that our constraints are useful in showing that tilings by certain polyhedra or combinations of polyhedra do not exist.

At a very late stage of our study, we found three more examples which allayed a concern that had developed. Up to Example 17, we had placed no points in our Figure 1 on the line $\mu_{E P}=3$, other than three having $\mu_{V E}=4$. As this line is a boundary of our space, we sought other examples. We found them by using the well-known monohedral tiling of space by the rhombic dodecahedron $D$ (with each facet being a rhombus whose long diagonal is $\sqrt{2}$ times its short diagonal); see [17]. Using the classical facet-to-facet tiling using $D$, we obtained $\left(\mu_{V E}, \mu_{E P}\right)=\left(\frac{16}{3}, 3\right)$, but improved on this by cutting $D$ into smaller convex polyhedra. Importantly these cuts did not hit a ridge-interior of $D$. Two other non facet-to-facet tilings resulted with $\left(\mu_{V E}, \mu_{E P}\right)=(8,3)$ and $\left(\mu_{V E}, \mu_{E P}\right)=(10,3)$. All three are plotted on Figure 1 as $18 \mathrm{a}, 18 \mathrm{~b}$ and $18 \mathrm{c}$.

ACKnowledgement: The second author was supported by the German research foundation (DFG), grant WE 1799/3-1. The first author's accommodation expenses, while visiting Jena for a period of joint research, came from the same grant.

\section{References}

[1] Cowan, R. (1978). The use of ergodic theorems in random geometry. Suppl. Adv. Appl. Prob. 10, 47-57.

[2] Cowan, R. (1980). Properties of ergodic random mosaic processes. Math. Nachr. 97, 89-102.

[3] Cowan, R. And Weiss, V. (2013). Graphical presentations of the 7-dimensional parameter space arising in tessellations of $\mathbb{R}^{3}$. Technical note available as document 85(a) on www-personal.usyd.edu.au/ rcowan/professional/randomgeom.html

[4] Kendall, W. S. and Mecke, J. (1987). The range of mean-value quantities of planar tessellations. J. Appl. Prob., 24, 411-421.

[5] Leistritz, L. And ZÄHle, M. (1992). Topological Mean Value Relationships for Random Cell Complexes. Math. Nachr. 155, 57-72.

[6] Mecke, J. (1984). Parametric representation of mean values for stationary random mosaics. Math. Operations. Statist. Ser. Statist. 15, 437-442.

[7] Miles, R. E. (1971). Poisson flats in Euclidean spaces. Part II: Homogeneous Poisson flats and the complementary theorem. Adv. Appl. Prob., 3, 1-43. 
[8] Møller, J. (1989). Random tessellations in $\mathbb{R}^{d}$. Adv. Appl. Prob. 21, 37-73.

[9] Nagel, W. And Weiss, V. (2008). Mean values for homogeneous STIT tessellation in 3D. Image Anal. Stereol., 27, 29-37.

[10] Nguyen, N. L., Weiss, V. And Cowan, R. (2013). Spatial tessellations derived from planar tessellations. In preparation.

[11] Okabe, A., Boots, B., Sugihara, K. And Chiu, S. N. (2000). Spatial Tessellations: Concepts and Applications of Voronoi Diagrams. 2nd. ed., Wiley, Chichester.

[12] RADECKE, W. (1980). Some mean-value relations on stationary random mosaics in the space. Math. Nachr. 97, 203-210.

[13] Schneider, R. And WeIL, W. (2008). Stochastic and Integral Geometry. Springer, Berlin Heidelberg.

[14] ThäLE, C. AND Weiss, V. (2010). New mean values for homogeneous spatial tessellations that are stable under iteration. Image Anal. Stereol. 29, 143-157.

[15] Weiss, V. and Cowan, R. (2011). Topological relationships in spatial tessellations. Adv. Appl. Prob. 43, 963-984.

[16] Weiss, V. And Z̈̈HLE, M. (1988). Geometric Measures for Random Curved Mosiacs of $\mathbb{R}^{d}$. Math. Nachr. 138, 313-326.

[17] Wells, D. (1991). The Penguin Dictionary of Curious and Interesting Geometry. Penguin Books, London.

[18] Ziegler, G. M. (2003). Face Numbers of 4-Polytopes and 3-Spheres. arXiv:math/0208073v2[math.MG]. 

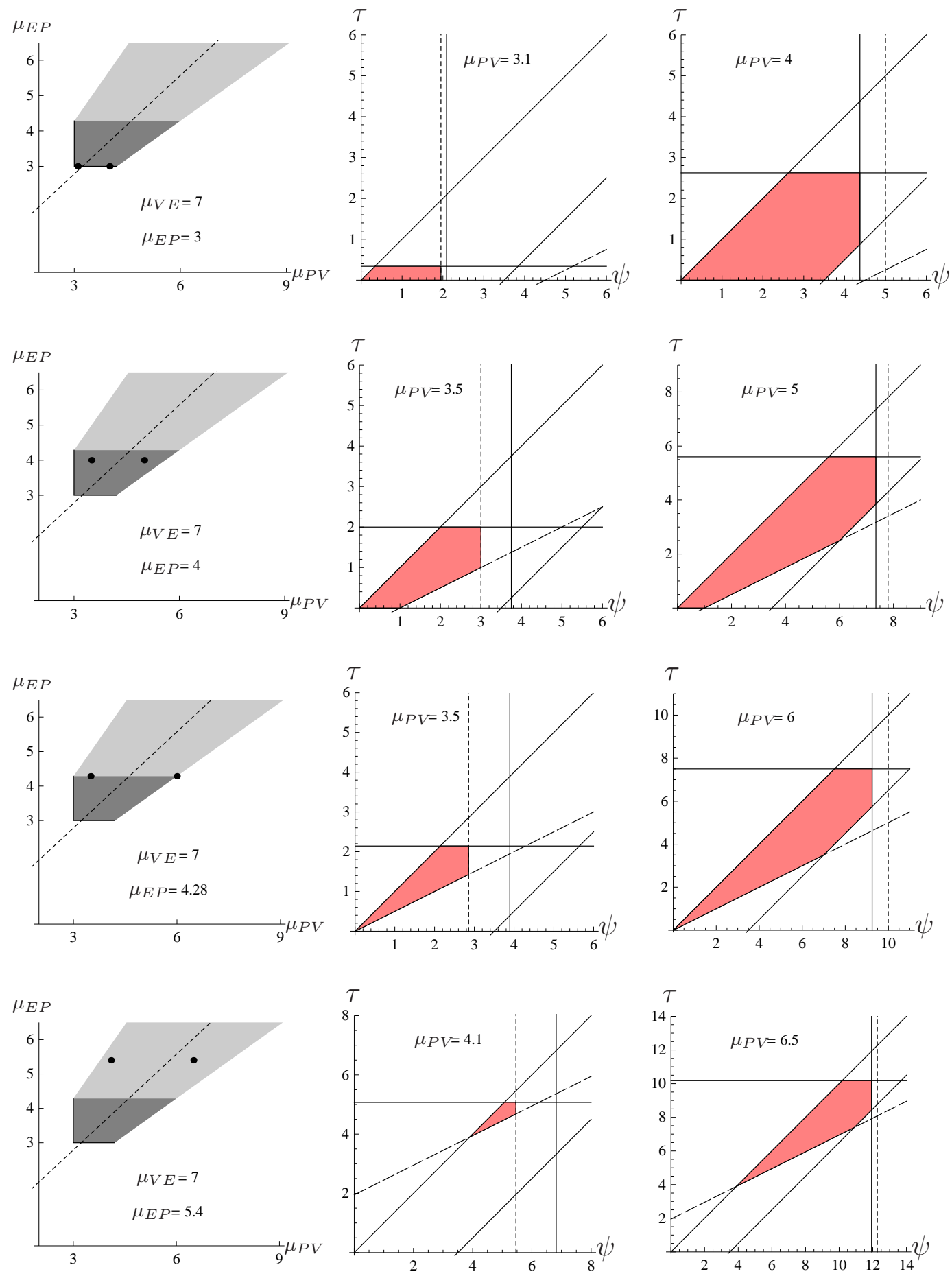

Figure 11: A diversity of shapes for the $(\psi, \tau)$ permitted domain when $\mu_{V E}=7$. The dots in the leftmost diagram of each row set the cyclic parameters for the two diagrams to its right. 